\title{
Physical Properties of a Molecular Cloud after a Penetrating Collision
}

\author{
Guillermo Arreaga-García* and Julio Saucedo-Morales
}

Departamento de Investigación en Física, Universidad de Sonora, Apdo. Postal 14740, C.P. 83000, Hermosillo, Sonora, Mexico

\begin{abstract}
This paper is aimed to present a set of numerical simulations of a penetrating collision, in which a small gas core (the bullet) gets into a larger core (the target). The gravitational collapse, in the target core, is supposed to be ongoing before the collision. After the collision, the integral properties of the resulting mixed gas core are calculated by using all the $S P H$ particles. Only three collision models are considered here according with their impact velocity, ranging from zero to Mach 15. The outcomes of these collision models are presented only for two values of the impact parameter $b$. Such collisions models allow to reveal how the occurrence of hydrodynamical instabilities favor the fragmentation of the cores in their central region, pointing out the possibility to have a lot of small cloudlets. We show hereby that penetrating collisions may have a major and favorable influence in the star formation process.
\end{abstract}

Keywords: Collision, collapse, hydrodynamics.

\section{INTRODUCTION}

By means of far-infrared and radio molecular observations, several authors [1,2] have made measurements of gas distributions in Orion, which clearly reveal the existence of two dissimilar gas clumps in close gravitational interaction. It is then possible that a large gas cloud be assembled by smaller gas elements by means of ineslastic collisions, in which a large structure is composed of many smaller and denser gas cores immersed in a more diffuse gas medium, see [3]. From the perspective of this formation mechanism, collisions are occurring between dissimilar neighboring gas cores which are all contained in the same larger gas structure [4].

The study of gas collisions has a long history from both theoretical and observational points of view. Even though it started back in the 1970s, the academic interest in this field has not entirely fallen off. Let it be mentioned, from the observational side, that a collision model consisting of two separated molecular elements colliding with each other was considered by [5] in order to propose an explanation for the triggering mechanism for the star formation process in the NGC 1333 system. The work of [6] is among the most recent papers considering a collision model of two structures in order to foresee the dynamical future of the Barnard 68 system.

The work of [7], who considered a model of the interstellar medium of a galaxy, in which the galaxy is thought to be formed by a discrete collection of molecular clouds, is mentioned from a theoretical perspective. Such a galaxy model was applied to simulate the interaction of the

*Address correspondence to this author at the Departamento de Investigación en Fisica, Universidad de Sonora, Apdo. Postal 14740, C.P. 83000, Hermosillo, Sonora, Mexico; E-mail: garreaga@ cifus.uson.mx
Large Magellanic cloud with the Small Magellanic cloud. Among their main conclusions they found that: (i) a very large number of collisions can occur between the molecular structures and that the most probable impact velocity $V_{i m p}$ and impact parameter $b$ are around the values $0-50 \mathrm{~km} / \mathrm{sec}$ and $0-2 R_{c}$, respectively, where $R_{c}$ is the initial radius of the colliding structure; (ii) the collisions of structures with unequal initial sizes can occur very frequently; (iii) it is possible in this galaxy model for a star cluster to be formed at the center of the colliding gas structure; (iv) the star formation efficiency strongly depends on the impact velocity and the impact parameter $b$.

Moreover, head-on collisions between two adjacent clouds were studied by [8], including several combination of parameters for the initial colliding clouds, such as size, average density and internal density structure (uniform or clumpy). The self-gravity of the gas was unfortunately not taken into account in their two-dimensional hydrodynamical calculations.

The studies performed by [7] and [8] have had an influence in the present paper, as we have attempted to take some of their ideas into account in order to improve their simulations. Besides, the present investigation can be considered as a continuation of the study carried out by [9] (hereafter Paper I). It must be emphasized that collision models of two gas cores, from a purely theoretical perspective with no intention of making any comparison with some observed systems, have been numerically studied in both Paper I and in the present paper.

It could be that collisions may also play an important role in star formation by altering the physical processes in cores, including their gravitational collapse, making it worthwhile to consider such collisions. It is currently known that most star forming molecular hydrogen gas clouds are mainly 
located in the arms of spiral galaxies, where cloud collisions may be very common in the Interstellar Medium.

Whether a collision does or does not help the fragmentation of the larger core is what this study aims to analyze. Consequently, some results which we consider noteworthy have been found in this investigation, such as the fact that the collapsed filament formed in the central region of the core fragments due to collisions. This is a very important result in star formation research, because each of the dense gas knots formed along the filament in the central core of the collapsing target core, could eventually form a proto-star. This result indicates that there are some collisions that have a major and favorable influence in the already initiated process of star formation. It is in these kind of collisions that the present study is mostly interested.

Generally speaking, two main possibilities are seen when there is a collision between two cores. One is that with a sufficiently high impact velocity the cores get dispersed entirely irrespective of their initial physical state. There is no chance for the gas to become clumpy in this case; the continuation of the star formation process is almost impossible. Another more interesting possibility for star formation is that the cores can remain or become gravitationally bound during the collision. This will happen if the collision has a sufficiently low impact velocity. The new mixed gas core formed by the colliding gas cores could be unstable against further gravitational collapse, then the mixed gas system may eventually collapse to produce new multiple proto-stellar clumps.

The impact velocity $V_{i m p}$ parameter space of two colliding identical cores was explored in Paper I, where we saw that the resulting gas configuration after the collision may still be forming gravitationally bound complex gas systems. These systems may include irregular filaments connecting gas clumps (the remnants of the original cores showing no evidence of interpenetration of the colliding cores) where it has been argued that the star formation process is still possible despite the occurrence of the collision.

The collision between two very dissimilar cores is studied in the present paper, considering different initial core sizes, masses and densities; nevertheless, it is focused on considering only low impact velocity collision as was the case in Paper I, but this time between a small core and a bigger core. As a result, it is possible to see that all the gas of the colliding cores somehow gets mixed giving rise to a new bound core, whose dynamical properties are going to be calculated. Mainly, the evolution of the thermal and rotational energy ratios with regards to the gravitational energy, denoted by $\alpha$ and $\beta$, respectively, are determined in the present paper. As is well known today, the outcome of a core undergoing gravitational collapse is mainly determined by these dynamical parameters.

The formation of hydro-dynamical instabilities in the interface region has been studied numerically in both Paper I and the present study. One of the main purposes of this paper is to investigate whether these hydro-dynamical instabilities may have an influence on the dynamics of the resulting mixed gas core and, more specifically, on its gravitational collapse process. This is remarkable, because a collision between cores can be a source of hydro-dynamical instabilities and, eventually, of turbulence in the interstellar medium.

It is noteworthy that [10] has also simulated cloud collisions (between two clouds), considering an impact velocity within the Mach $25-35$ range and that dissimilar collision models were also considered by [11]. But all their pre-collision clouds are initially in hydrodynamical equilibrium since their were modeled as Bonnor-Ebert spheres.

It must finally be emphasized that both Paper I and the present study, are devoted to studying collisions with cores with a clear initial tendency for gravitational collapse prior to collision, allowing us to uncover the formation of collapsing clumps by fragmentation. This observation has made an important difference from the results derived from simulations reported by [10] and [11].

The outline of this paper is as follows. The particle distribution that represents the initial target core, which will be involved in all the subsequent collisions, is described in Sections 2 and 2.1. The geometry of the collision models is described in Section 3. The parameters chosen for the evolution code Gadget2 are described in Section 3.2. The time evolution of the most important features of these simulations by means of 2D iso-density plots (although the collision events are fully 3D), and the collision systems's integral properties taken into consideration are both reported in Section 4. The relevance of these results in view of those reported in previous works is discussed in Section 5. Finally, some concluding remarks are made in Section 6.

\section{THE INITIAL PHYSICAL STATE OF THE COLLIDING CORES.}

We consider two dissimilar spherical cores made of molecular hydrogen gas. The cores are face to face, as is illustrated in Fig. (1). The bigger core acts as the target core and it is initially motionless in space. The smaller core plays the role of the bullet as it onrushes the target core with an initial translational velocity along the $Y$ axis. It should be noted that each core rotates as a rigid body around its own vertical axis in the counterclockwise direction. Both cores have a uniform radial density profile.

\subsection{Initial Kinematics}

The target core has a radius $R_{0}=3.0138 \times 10^{16} \mathrm{~cm}$ and mass $M_{0}=2.21 M_{\odot}$. This core is similar (although not identical) to that described by [12] and also considered in Paper I. The average density of this spherical core is $\rho_{\text {ave }}=3.8 \times 10^{-17} \mathrm{~g} / \mathrm{cm}^{3}$, so that, it will simply be called a core, according to the classification scheme given by [13] in his Table $\mathbf{1 .}$ 


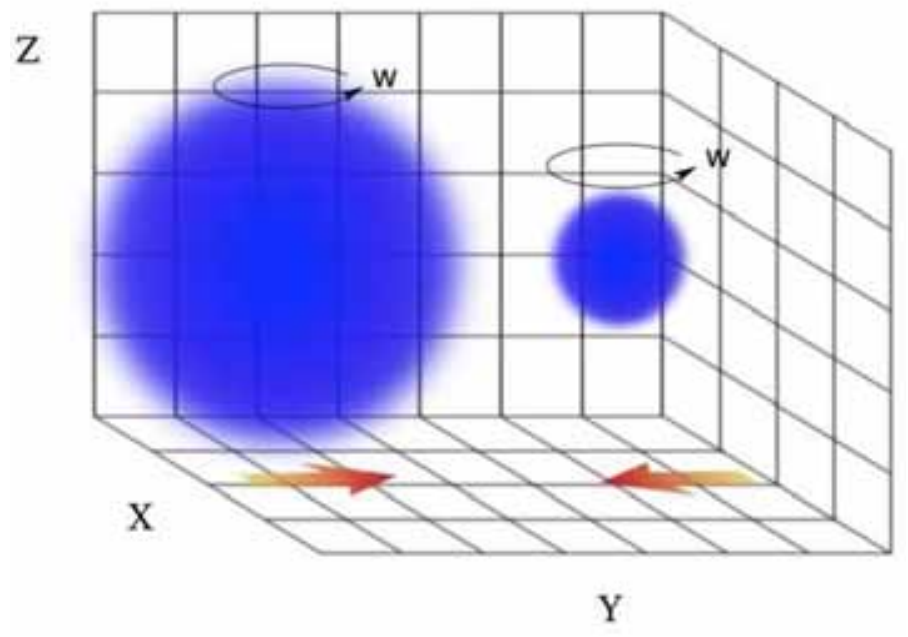

Fig. (1). The initial geometry of the colliding clouds.

Table 1. Density and time normalizing scales.

\begin{tabular}{|c|c|c|}
\hline$\rho_{0}$ & $t_{f f c}$ & $t_{f f}$ \\
\hline \hline $1.6 \times 10^{-22} \mathrm{gr} / \mathrm{cm}^{3}$ & $5.8 \times 10^{13} \mathrm{sec}=1.846 \mathrm{Myr}$. & $1.64 \times 10^{14} \mathrm{sec}=5.22 \mathrm{Myr}$. \\
\hline
\end{tabular}

It is relatively easy to observe these small cores as isolated systems in low mass star forming regions, such as Taurus, see [14]. If cores like these where located within a more dense gas environment, such as the Orion cluster, then we expect they would have already had multiple collisions.

The mass of the onrushing core is only $M_{O C}=0.09 M_{\odot}$, which is very small compared with the target core mass. As the size of this bullet core is $R_{0} / 4$. As was the case with the target core, the bullet core can also be identified with a small and diffuse core according to Table 1 of [13]. Furthermore, the density contrast between the colliding cores is $\rho_{\text {bullet }} / \rho_{\text {target }}=2.6$. As the onrushing core is almost three times denser than the target core, the interpenetration of cores will be expected, as it will be further observed.

The cores involved in the collision events more frequently observed, are most likely typical giant molecular cores with a radius around 40 parsecs and a mass around $10^{5} M_{\odot}$. Despite this observational fact, we have chosen to model the penetrating process of a tiny core into an small core, because we would like to see whether these collisions are possible mechanisms favoring low mass multiple systems formation.

As previously mentioned, the target core has no translational velocity, but it is rigidly rotating, so that the initial rotational velocity of the $i-t h$ particle is given by $\vec{v}_{i}=\vec{\Omega}_{0} \times \vec{r}_{i}=\Omega_{0}\left(-y_{i}, x_{i}, 0\right)$, where we have taken $\vec{\Omega}_{0}=\Omega_{0} \times \vec{k}$ with $\Omega_{0}$ being the constant magnitude of the initial angular velocity and $\hat{\mathrm{k}}=(0,0,1)$.
Besides, a solid rotation and a finite temperature of the onrushing core have also been provided. Therefore, in a kinematical sense, the onrushing core can be considered to be formed by the core of the target core, as both cores share the following values of angular speed and sound speed:

$c_{0} \equiv 30363.1 \mathrm{~cm} \mathrm{~s}^{-1}$,

$\Omega_{0} \equiv 5.969 \times 10^{-14} \mathrm{~s}^{-1}$.

As shall be seen in Section 2.2, the reason for choosing these values of $c_{0}$ and $\Omega_{0}$ is related to the initial energies given to the target core. However, these values of angular speed and sound speed for the onrushing core are not significant in determining the fate of the colliding system, as we will see later.

\subsection{Virial Relations and Initial Core Energies}

It has been argued, from both the theoretical and the observational sides, that molecular cores are not likely to be in thermodynamical equilibrium. If they were, then the core energies would satisfy the following virial relation:

$2\left(E_{\text {therm }}+E_{\text {kin }}\right)+E_{\text {grav }}=0$

where $E_{\text {therm }}$ is the average total thermal energy; $E_{k i n}$ is the total kinetic energy and $E_{\text {grav }}$ is the total gravitational energy. The relation given in Eq. (2) can be re-written in the equivalent form

$\alpha+\beta=\frac{1}{2}$, 
where the thermal and kinetic energy ratios with respect to the gravitational energy, denoted by $\alpha$ and $\beta$, respectively, have been introduced.

Since the target core is rotating as a rigid body, the ratio between the rotational energy and the gravitational potential of the isolated core, can also be defined, analogously by $\beta$, $\beta_{r}$, see Eq. (4) below. According to the gravitational collapse literature, the dynamical properties of collapsing and rotating cores are commonly characterized by means of their thermal and rotational energy ratios, $\alpha$ and $\beta_{r}$.

The values $c_{0}$ and $\Omega_{0}$ considered in this paper, have been chosen so that the energy ratios for the target core have the following initial values:

$\alpha \equiv \frac{E_{\text {therm }}}{\left|E_{\text {grav }}\right|}=0.26$

$\beta \equiv \frac{E_{\text {kin }}}{\left|E_{\text {grav }}\right|}=0.16$

$\beta_{r} \equiv \frac{E_{\text {rot }}}{\left|E_{\text {grav }}\right|}=0.16$

It is important to remind that these selected values of $\alpha$ and $\beta_{r}$ are known to favor the collapse of the target core in an isolated system.

The values of the $\alpha$ and $\beta$ ratios during the collision will be calculated in order to compare the actual values with those given by the virial relation Eq. (3). $\beta_{r}$ will also be calculated later in order to measure how much the colliding system departs from the pure target core rotational state, see Section 3.4.

A density perturbation on the initial particle distribution of each core was implemented in such manner that, at the end of the gravitational collapse of the target and the onrushing cores, a binary system formation might be the final outcome. This perturbation is applied to the mass of each particle $m_{i}$ according to:

$m_{i}=m_{0}\left(1+\operatorname{acos}\left(m \phi_{i}\right)\right)$,

where $m_{0}$ is the unperturbed mass of the simulation particle, the perturbation amplitude is set to $a=0.1$ and the mode is fixed to $m=2 . \phi$ is the azimuthal angle of each particle with respect to the coordinate system based on the mass center of each core. The same was done in Paper I and in previous papers about collapse, see $[15,16]$, We have found that this mass perturbation is almost irrelevant in the core collision models scenario; although, it is very important in the filament formation as a result of the collapse in the isolated core, as it will be appreciated in Section 4.1.

\section{COLLISION MODELS AND COMPUTATIONAL CONSIDERATIONS}

In the present study a total set of $N_{p}=5,170,680$ particles generated the two initial cores by a traditional Monte Carlo scheme, in which the particles are located randomly in the volume space, as has already been previously described by [9]. 4,967,711 were used to generate the target core and and 202,969 for the onrushing core. The average mass of a simulation particle (after having applied the mass perturbation described by Eq.(5)) is $m_{p}=M_{t} / N_{p} \equiv 4.47 \times 10^{-7} M_{\odot}$, where $M_{t}$ is the total mass contained in the simulation box, that is, including both the mass of the target and the onrushing clouds.

The mass center (MC) initial position (in rectangular coordinates $(X, Y, Z))$ of the target core is located at $\vec{X}_{T C}=\left(0,-R_{0}, R_{0}\right)$ while the MC for the onrushing core is located at $\vec{X}_{O C}=\left(0, R_{0}, R_{0}\right)$. As previously mentioned, $R_{0}$ is the initial radius of the target core, see Fig. (1).

The approach velocity $V_{a p p}$ (or impact velocity) is defined as the translational velocity given to all the particles of the bullet core, in such a manner that its MC moves with the velocity $\vec{V}_{O C}=\left(0,-V_{a p p}, 0\right)$. As it was mentioned, the target core MC is initially at rest $\vec{V}_{T C}=(0,0,0)$.

\subsection{Time and Density Scales}

The impact velocity $V_{a p p}$ speeds up the evolution and the subsequent collapse of the cores, so that the evolution of the colliding system is expected to be faster than the collapse of the isolated core left by itselft under the influence of its own gravitational force.

A physically motivated time scale for describing these kind of collision models is the crossing time, which can be defined as $t_{c r}=R_{0} / V_{a p p}$. Other density and time scales for describing the core system evolution considered in this paper are in Table 1. This new scale is useful for comparing the results of these simulations with those already reported in Paper I.

It should be clear that the density and times scales shown in Table 1, are useful just as normalizing factors for the forthcoming evolution plots. These scales do indeed have a clear physical meaning for the models studied in Paper I. For instance, for model $\mathrm{HO}-1$, the crossing time is $t_{c r}=$ $1.16 \times 10^{14} \mathrm{sec}$ while for model $H 0-2$, we have $t_{c r}=5.82 \times 10^{13} \mathrm{sec}$.

It can be appreciated that for these models the crossing time is, at least, of the same order as the time scale defined in Table $\mathbf{1}$, that is $t_{c r} \leq t_{f f}$. Therefore, both time scales, $t_{c r}$ and $t_{f f}$, are equally suitable for describing the dynamics of our colliding systems.

\subsection{The Evolution Code}

The fully parallelized Gadget 2 code is used in the present paper, which implements the $S P H$ (smooth particle hydrodynamics) technique; see [17] for a detailed description of the code. The Gadget 2 code is based on the tree - PM method for computing the gravitational forces and on the standard SPH method for solving the Euler equations of hydrodynamics. The following standard features are incorporated by Gadget2: (i) each particle $i$ has its own smoothing length $h_{i}$; (ii) the particles are also allowed to have individual gravitational softening lengths $\varepsilon_{i}$, whose values are adjusted in such a manner that for every 
Table 2. Collision models.

\begin{tabular}{|c|c|c|c|c|}
\hline Model & $\frac{b}{R_{0}}$ & $\frac{V_{\text {app }}}{c_{0}}$ & $\log _{10}\left(\frac{\rho_{\max }}{\rho_{0}}\right)$ & $\frac{t_{\max }}{t_{f f}}$ \\
\hline \hline HO-0 & 0.0 & 0.0 & 10.35 & 1.95 \\
\hline HO-1 & 0.0 & 7.69 & 5.27 & 1.86 \\
\hline HO-2 & 0.0 & 15.39 & 7.82 & 1.84 \\
\hline Ob-0 & $\frac{1}{2}$ & 0.0 & 5.19 & 1.95 \\
\hline Ob-1 & $\frac{1}{2}$ & 7.69 & 4.33 & 1.77 \\
\hline
\end{tabular}

time step $\varepsilon_{i} h_{i}$ is of order unity; (iii) Gadget2 fixes the value of $\varepsilon_{i}$ for each time-step using the minimum value of the smoothing length of all particles, that is, if $h_{\min }=\min \left(h_{i}\right)$ for $i=1,2 \ldots N$, then $\varepsilon_{i}=h_{\text {min }}$.

As the particles interact in the collision system by means of viscosity, there is a contribution from a viscosity term included in the momentum equation of motion. It is thus very important to specify some details about the Gadget 2 implementation of a Monaghan-Balsara form for the artificial viscosity, see [18 and 19]. The strength of the viscosity is regulated by setting the parameter $\alpha_{v}=0.75$ and $\beta_{v}=\frac{3}{2} \times \alpha_{v}$, see Equation (14) in [17]; we have fixed the Courant factor to 0.1 .

\subsection{Equation of State and Resolution}

The barotropic equation of state proposed by [20] is used to take into account the heating of the gas due to both core contraction and energy dissipation from artificial viscosity. Thus, the following equation of state was used in this paper to carry out all the simulations:

$p=c_{0}^{2} \rho\left[1+\left(\frac{\rho}{\rho_{\text {crit }}}\right)^{\gamma-1}\right]$

where $\gamma \equiv 5 / 3$ and the critical density has the fixed value $\rho_{\text {crit }}=5.0 \times 10^{-14} \mathrm{~g} \mathrm{~cm}^{-3}$.

A wave front is formed at the cores' interface when the collision takes place, as the kinetic energy is transformed into thermal energy by the artificial viscosity introduced in the evolution code (see Section 3.2). However, the cooling time $\tau_{\text {cool }}$ is much shorter than both the local sound crossing time and the free fall time, so the isothermal condition can be safely used for the wave front region, as will be shown in the following.

The density in the thin dissipative region for a strong adiabatic shock, from the Rankine-Hugoniot jump condition, is about four times the pre-shock density; consequentely, the post-shock number density is expected to be about $n_{p s} \approx 100 \mathrm{~cm}^{-3}$. Following [21], the cooling time can be estimated from the expression

$\tau_{\text {cool }} \sim \frac{2.4 \times 10^{5}}{n_{H}} y r s$.

This was obtained by using the estimates for the wave front region that $\tau_{\text {cool }} \sim 2400$ yrs $=0.0024$ Mys.

The local sound crossing time for the bullet and target cores are $\tau_{\text {cross }} \sim R_{0} / c_{0} \sim(7-28) M y r$, respectively; while the evolution times required to describe the collapse of the collision system are in the range 7-10 Myrs, as it can be seen in Section 3, particularly in Tables $\mathbf{1}$ and $\mathbf{2}$. Therefore, it can be assumed that the wave front remains isothermal as $\tau_{\text {cool }}<<\tau_{\text {cross }}$ and $\tau_{\text {cool }}<<t_{f f}$. On the other hand, the collisions between clumps can be considered to be inherently inelastic, as the kinetic energy of the collision can then be efficiently radiated away by waves, see [22].

Following [23], the smallest mass that an $S P H$ calculation can resolve is $m_{r} \approx M_{J} /\left(2 N_{\text {neigh }}\right)$, where $N_{\text {neigh }}$ is the number of neighboring particles included in the $S P H$ kernel and $M_{J}$ is the spherical Jeans mass defined by

$M_{J} \equiv \frac{4}{3} \pi \rho\left(\frac{\lambda_{J}}{2}\right)^{3}=\frac{\pi^{\frac{5}{2}}}{6} \frac{c^{3}}{\sqrt{G^{3} \rho}}$.

Then, the mass particle $m_{p}$ must satisfy $m_{p} / m_{r}<1$ for the collision models to comply with the Jeans requirement from [24]. If the instantaneous sound speed is approximated by $c=\sqrt{p / \rho}$, according to Eq.(6), $M_{J}$ is given by:

$M_{J}=\frac{\pi^{\frac{5}{2}}}{6} \frac{c_{0}^{3}}{\sqrt{G^{3} \rho}}\left[1+\gamma\left(\frac{\rho}{\rho_{\text {crit }}}\right)^{\gamma-1}\right]^{\frac{3}{2}}$.

Considering that a model reaches a peak density $\rho_{\text {max }} \approx 3 \times 10^{-11} \mathrm{~g} \mathrm{~cm}^{-3}$, we find that the minimum Jeans mass is given by $\left(M_{J}\right) \approx 7.6 \times 10^{-4} M_{\odot}$, from which we obtain the ratio $m_{p} / m_{r}=0.05$. Therefore, the Jeans resolution requirement is satisfied quite easily for this model. 


\subsection{Calculation Procedure for Measuring the Physical Properties}

The way the gas core integral properties were calculated shall now be described. The first step is to read the density and the gravitational potential for every particle $i=1 \ldots N_{t}$ in the simulation from the snapshots produced by the Gadget 2 code. Secondly, the sum is made over all the $N_{t}$ particles to obtain the thermal energy of the system, that is

$E_{\text {therm }}=\sum_{i=1}^{N_{t}} \frac{3}{2} \frac{P_{i}(\rho) m_{i}}{\rho_{i}}$,

where $P_{i}$ is the pressure associated with the particle $i$, with density $\rho_{i}$ and mass $m_{i}$, by means of the equation of state given in Eq. (6).

The calculation of the kinetic energy is also straightforward, as it was the case for the thermal energy. Nevertheless, a word of explanation is required to calculate the system's rotational energy $\beta_{r}$, as this must be a parameter that is meant to be useful for measuring the disturbance of the rotating mixed gas core with respect to the rotational state of the initial target core. It should indeed be defined relatived to the $Z$-axis of the target core itself. Let $\vec{x}_{i}$ and $\vec{v}_{i}$ be the position and velocity of particle $i$ with respect to the coordinates origin located between the collapsing cores, as it can be seen in Fig. (1).

As a result, the coordinates of particle $i$ with respect to a system of coordinates fixed at the MC of the target core would be ${\overrightarrow{x^{\prime}}}_{i}=\vec{x}_{i}-\vec{X}_{T C}$, where $\vec{X}_{T C}=\left(0,-R_{0}, R_{0}\right)$, as previously mentioned. The velocities are the same in both systems of coordinates. Then the azimuthal angle $\phi$ associated with the rotation of each particle $i$ with respect to this $Z$-axis, that is $\phi_{i}$, can be calculated by taking the product $\phi_{i}=\arctan \left({\overrightarrow{x^{\prime}}}_{i} \cdot \hat{\jmath} / \vec{v}_{i} \cdot \hat{\imath}\right)$, where the unit vectors are $\hat{\imath}=(1,0,0)$ and $\hat{\jmath}=(0,1,0)$.

Afterwards, the rotational energy can be estimated by taking the projection of the velocity of the particle along the unit azimuthal vector $\hat{e}_{\phi_{i}}=-\sin (\phi) \hat{\imath}+\cos (\phi) \hat{\jmath}$, that is

$E_{\text {rot }}=\sum_{i=1}^{N_{t}} \frac{1}{2} m_{i}\left(\vec{v}_{i} \cdot \hat{e}_{\phi_{i}}\right)^{2}$
$E_{\text {kin }}=\sum_{i=1}^{N_{t}} \frac{1}{2} m_{i} \vec{v}_{i} \cdot \vec{v}_{i}$.

where it has also been shown that the kinematic energy is obtained directly by means of a single sum over all the simulation particles.

\section{RESULTS}

Although the collisions are clearly a $3 D$ phenomenon, showing the main simulation results using $2 D$ iso-density plots for a thin slice of matter parallel to the $X Y$ plane will be attempted. Some $2 D$ plots with arrows indicating the velocity fields of the particles have also been included.
The procedure to illustrate the results with $2 D$ iso-density plots is as follows. The $S P H$ particle with the maximum density in the entire volume space of a simulation is located. The vertical height of the thin slice of material is determined and denoted by $z_{\max }$. The width of the slice, centered on the $Z_{\max }$ coordinate, is determined in order to have about $10,000 S P H$ particles entering into the slice. A color scale to distinguish the iso-density curves is set once the $S P H$ particles defining the slice have been selected.

This procedure for building $2 D$ iso-density plots was used to display the evolution of both the target core as an isolated system and the results of the collision systems as well. Looking at the electronic version of this paper is recommended, zooming in on the plots to truly appreciate the details of these two types of figures with iso-density curves or velocity fields.

It should be noted that there is no relation between the density colors associated with different panels, not even in the same figure.

\subsection{The Collapse of the Target Core}

The temporal evolution of the target core, considered as an isolated system evolving under the influence of its own gravitational force, will be briefly described in this section.

The evolution of a uniform density core has been reproduced by many research groups around the world using different codes based on both grids and particles, see for instance [25] and [26] and the references therein. The uniform model results in $[15,16]$ have been successfully reproduced, from which the reliability of our calculations for evolving the collapse of cores with the Gadget 2 code is established, as the collapse process until peak densities of the order of $3.0 \times 10^{-11} \mathrm{~g} \mathrm{~cm}^{-3}$ has been followed. The same Gadget 2 code has been used to evolve the initial conditions in the present study, to follow the collision process.

The numerical simulations performed so far have proved that an isolated rotating core contracts itself to an almost flat configuration approximately within a free-fall time of a dynamical evolution. However, the Monte Carlo method described in Section 2 for constructing the initial core, produces an initial density and velocity distribution of the particles with a peculiar behavior, which means that the core starts its evolution with an spatial expansion so that the peak density of the core decreases by up to two orders of magnitude.

As expected on the basis of previous research and those of other groups elsewhere, it has also been observed in both Paper I and the present paper, that the evolution of a uniform density core determined by means of the Monte Carlo method does indeed collapse, though with some delay, in such a manner that the core begins to lengthen while forming a well-defined filament that is surrounded by a halo, still having cylindrical symmetry, as it can be seen in Fig. (2). 


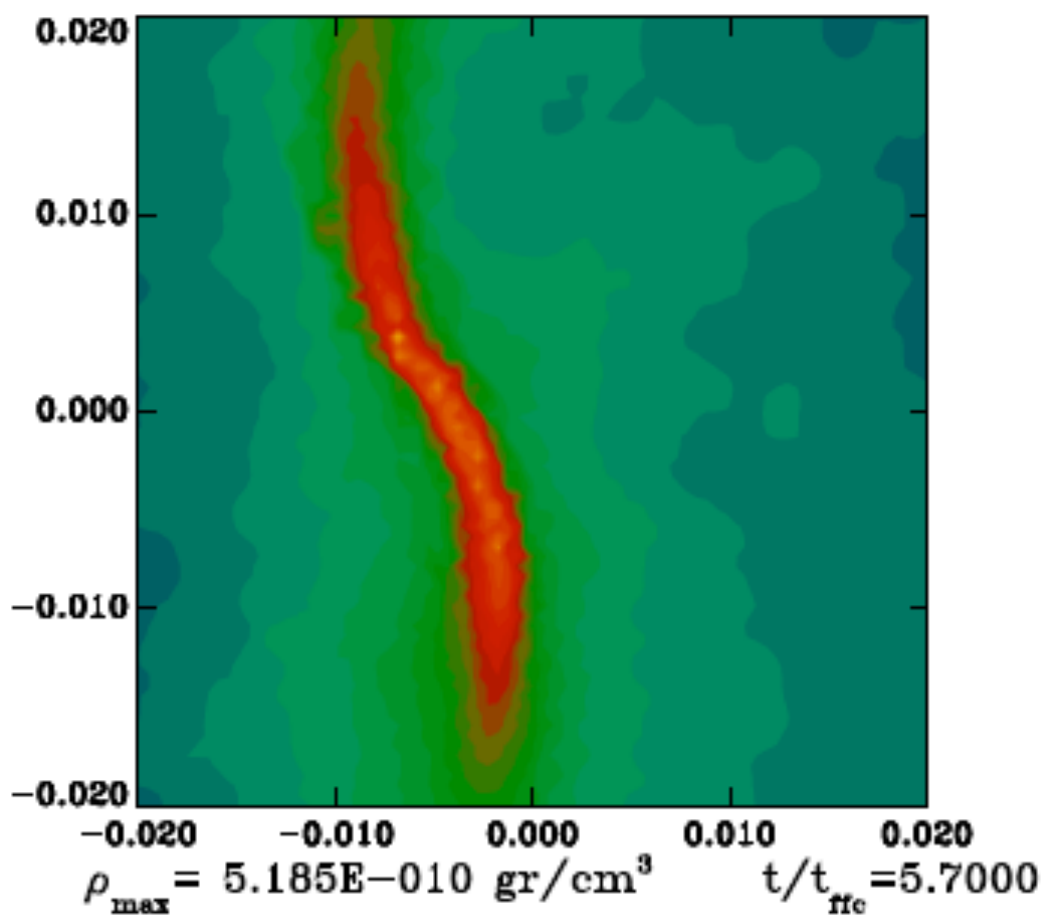

Fig. (2). Iso-density plot for a thin slice of matter parallel to the $X Y$ plane illustrating the gravitational collapse of the isolated and rotating cloud, which we shall make collide later.

Thanks to the mass perturbation given in Eq. (5), we have reached this resultant filament. It must be noticed that the filament developing in the central region of the collapsed core does not reveal any sign of fragmentation, at least until the peak density of $6.0 \times 10^{-10} \mathrm{~g} / \mathrm{cm}^{3}$ is reached.

The results of the collision models will be described in later subsections, in all of which there is a clear trend to collapse, as can be seen in Fig. (3).

\subsection{The Models $\mathrm{HO}-\mathrm{O}$ and $\mathrm{Ob}-\mathbf{0 \text { . }}$}

In these models the bullet core falls freely in the gravitational field of the target core. The target core completely engulfs the bullet, as can be appreciated in Figs. (4 and 5). There is no important difference in the results observed for model $\mathrm{HO}-0$ and $O b-0$, because the latter has a small impact parameter.

A small slab of gas forms when the particles collide, which can be seen as a filament in the interface between the cores. Nevertheless, no significant increase in the peak density of these simulations is observed, as can be seen in Fig. (3). Neither does the wave front cause geometric distortion, as the target core continues with its rotational motion.

One effect of the collision is to promote the occurrence of fragmentation in the central region of the mixed core. This observation should be contrasted with the target core evolution as an isolated system (see Fig. 2), which never shows any tendency to fragmentation on the filament developed in the central region of the core.

\subsection{The Models $H O-1$ and $O b-1$.}

During the initial stage of the the spatial expansion of the target core, there is a sudden increase in the peak density due to the gas slab formed in the region of interface with the first colliding particles, as can be seen in Fig. (3). After the first contact with the colliding particles, the peak density does decrease again, as was to be expected from the standard hydrodynamic theory. The simulations' peak density rises again for the subsequent evolution; although this time guided instead by the normal gravitational collapse of the mixed gas core formed after the collision. A bow is formed by the gas slab in the interface, whose arms grow in the outward direction. Meanwhile, the densest region of the onrushing core is still digging deep inside the target core, as can be seen in Figs. (6 and 7).

A re-distribution of the densest gas mass in the central region of the mixed gas core has been provoked by the penetration of the bullet into the target core. As can be seen in the last panels of Figs. (6 and 7), the original circular motion of the target core has almost disappeared, at least in the new core central region. This structural change is more evident in the model $H O-1$ than in the model $O b-1$. A development of small gas clumps was observed in a turbulent environment in the former model, while in the 

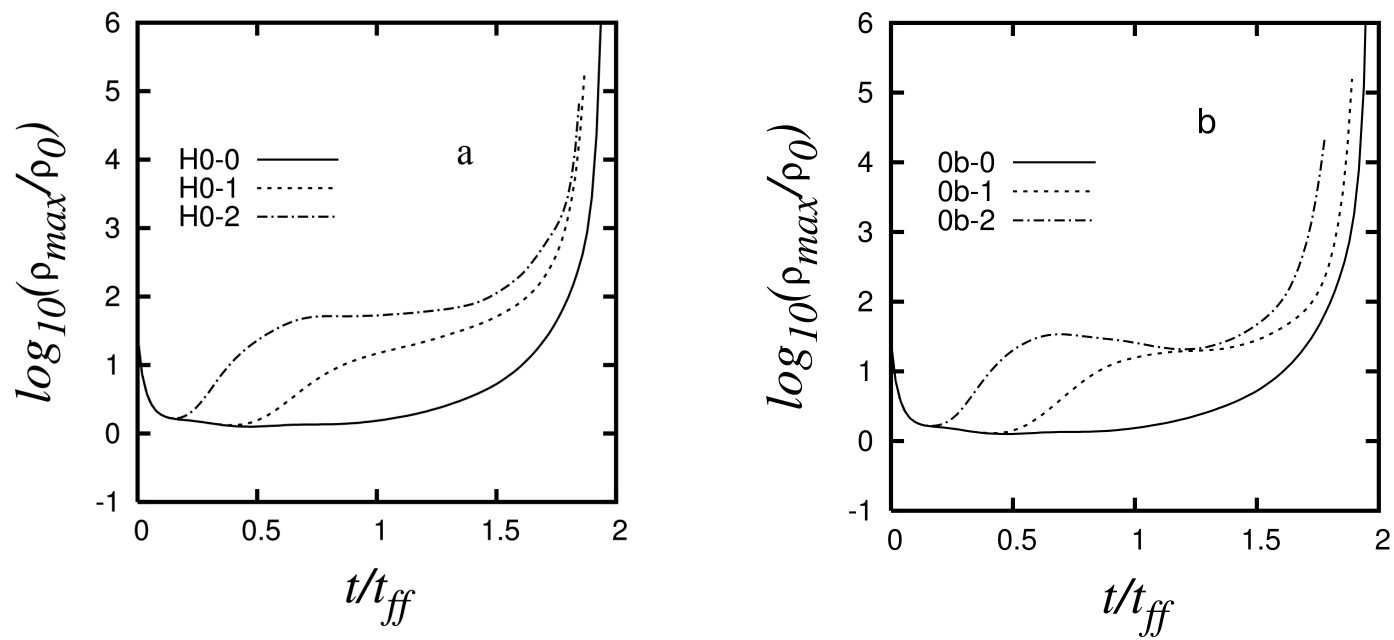

Fig. (3). Time Evolution of peak densities for (left) head-on collisions and (right) oblique collisions.
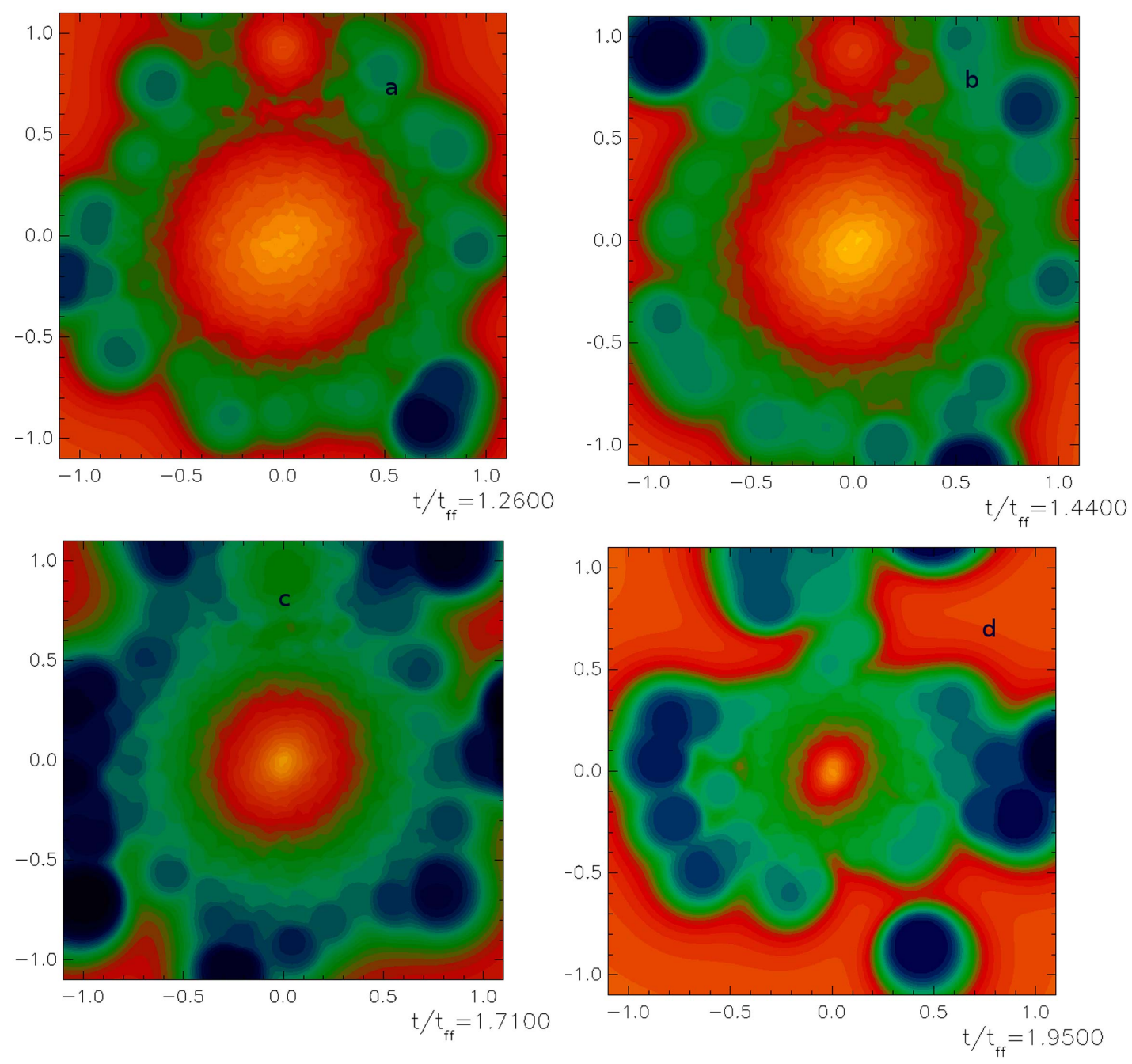

Fig. (4). Iso-density plot for a thin slice of matter parallel to the $X Y$ plane illustrating the collision process of simulation H0 0. 

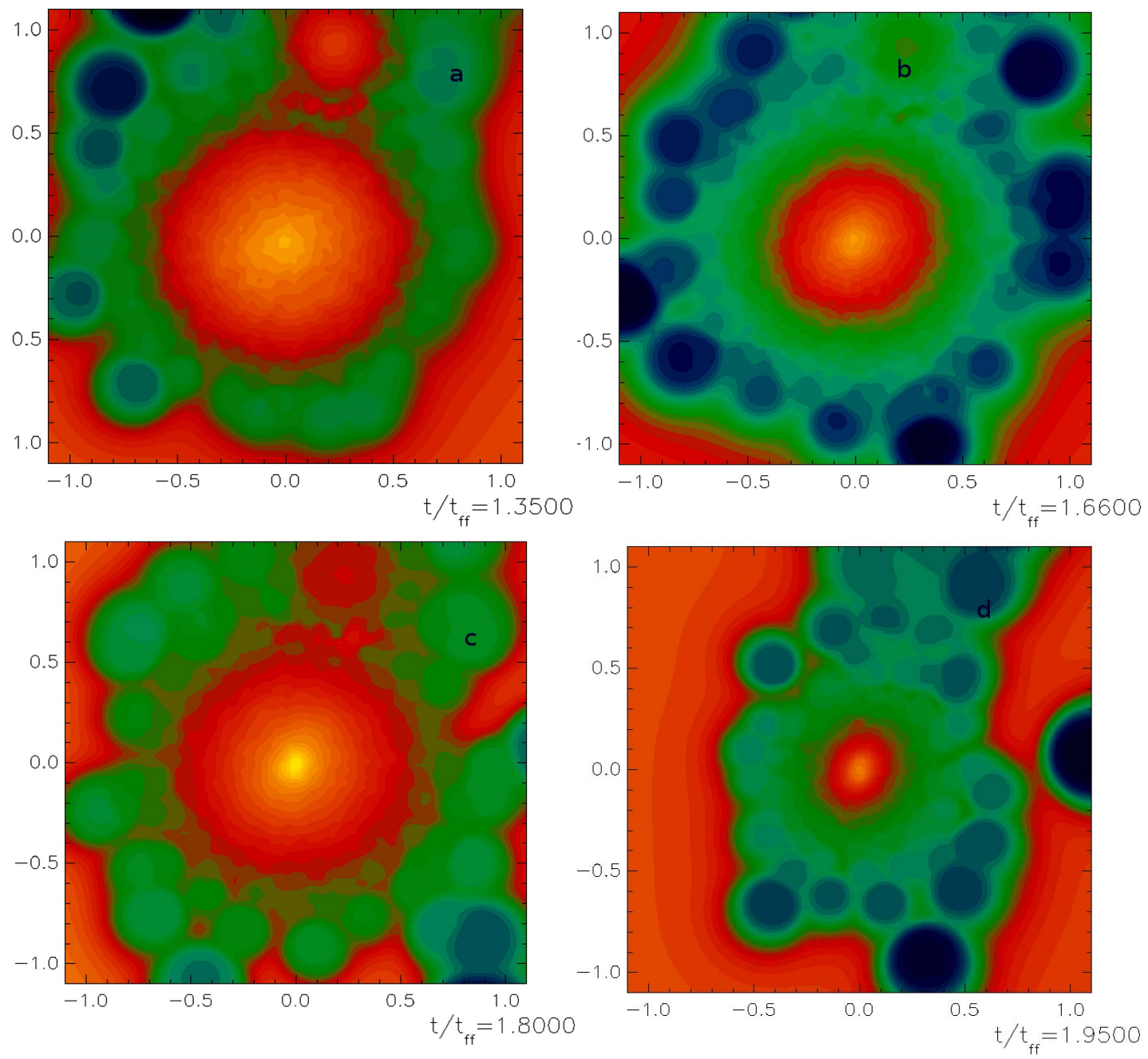

Fig. (5). Iso-density plot for a thin slice of matter parallel to the $X Y$ plane illustrating the collision process of simulation $0 \mathrm{~b} \sim 0$.
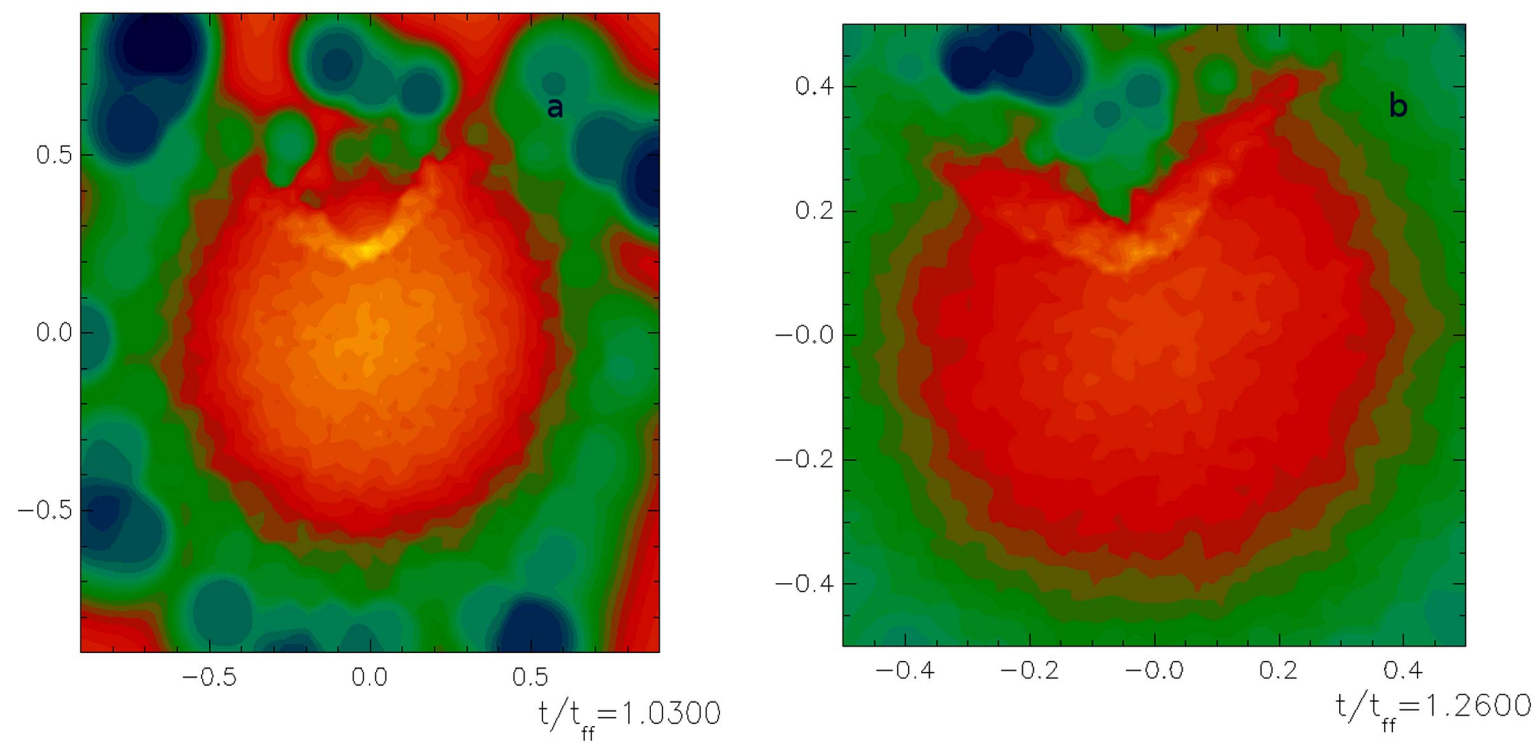

Fig. (6). Contd... 

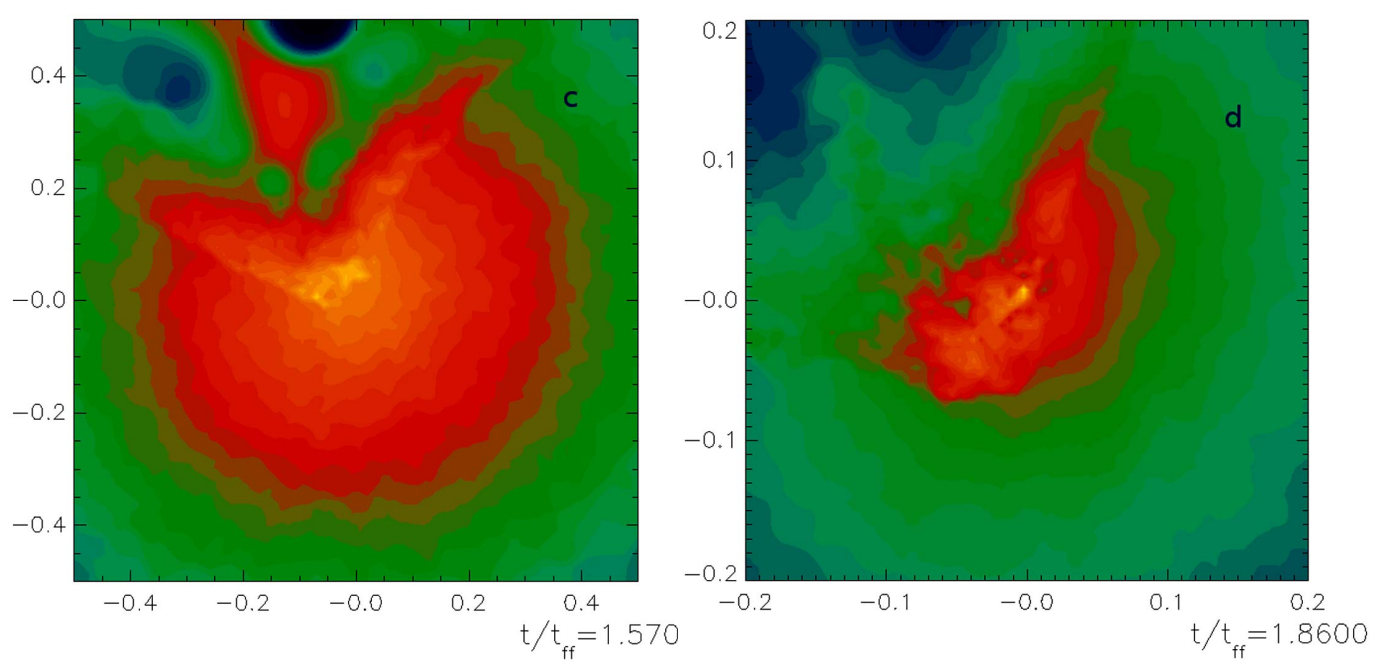

Fig. (6). Iso-density plot for a thin slice of matter parallel to the $X Y$ plane illustrating the collision process of simulation H0 1.
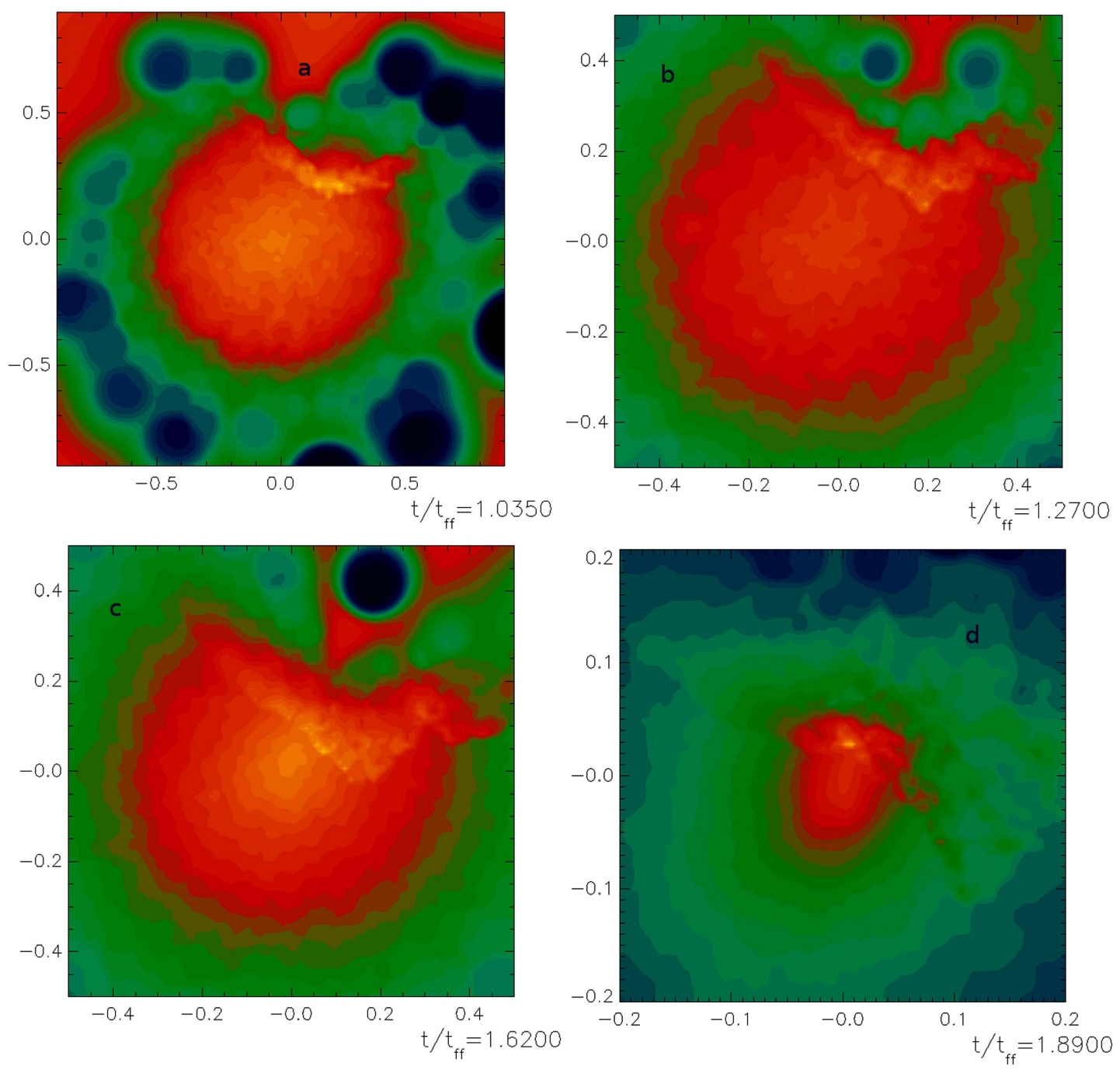

Fig. (7). Iso-density plot for a thin slice of matter parallel to the $X Y$ plane illustrating the collision process of simulation $0 \mathrm{~b} \sim 1$. 

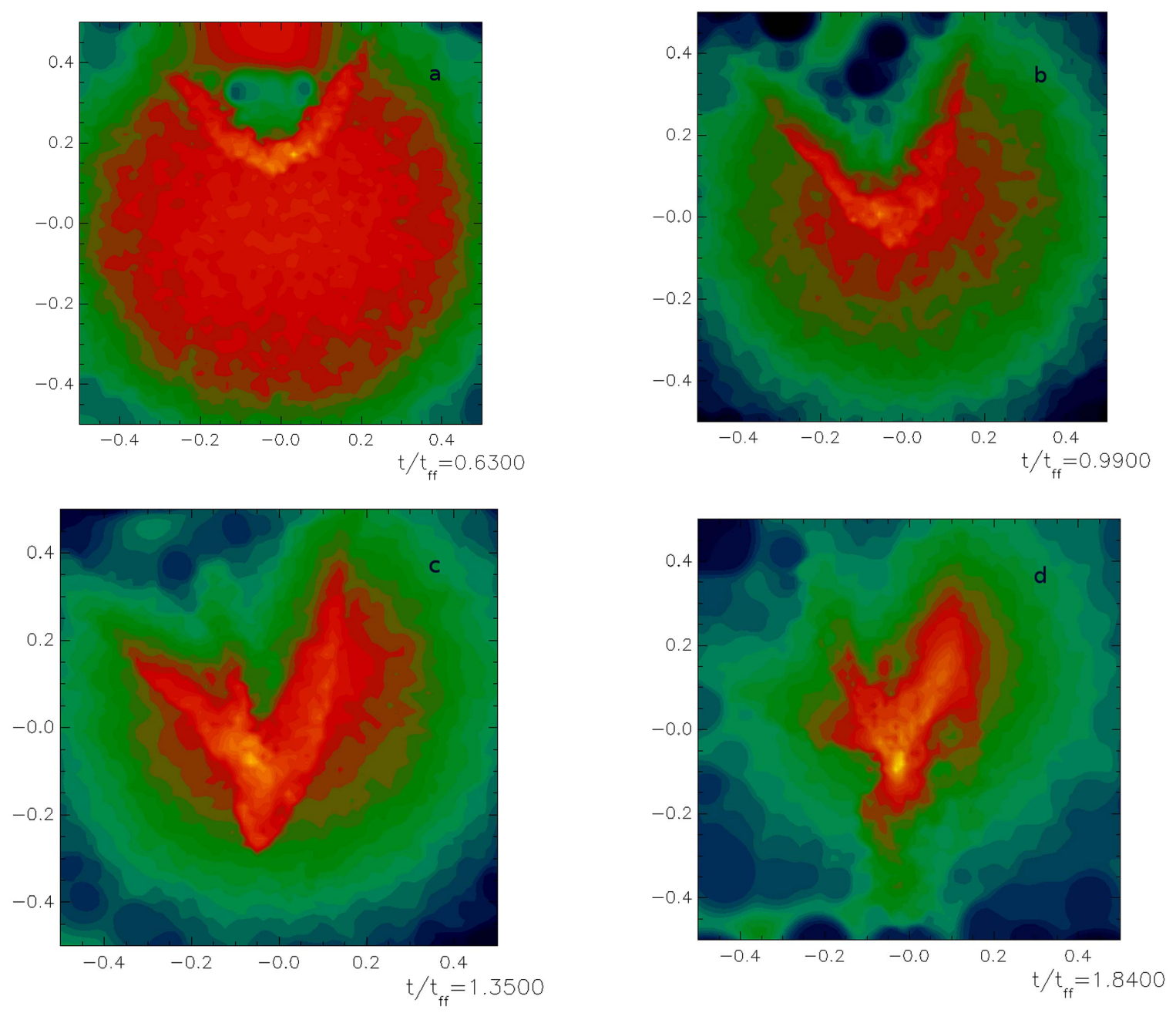

Fig. (8). Iso-density plot for a thin slice of matter parallel to the $X Y$ plane illustrating the collision process of the simulation H0 2.
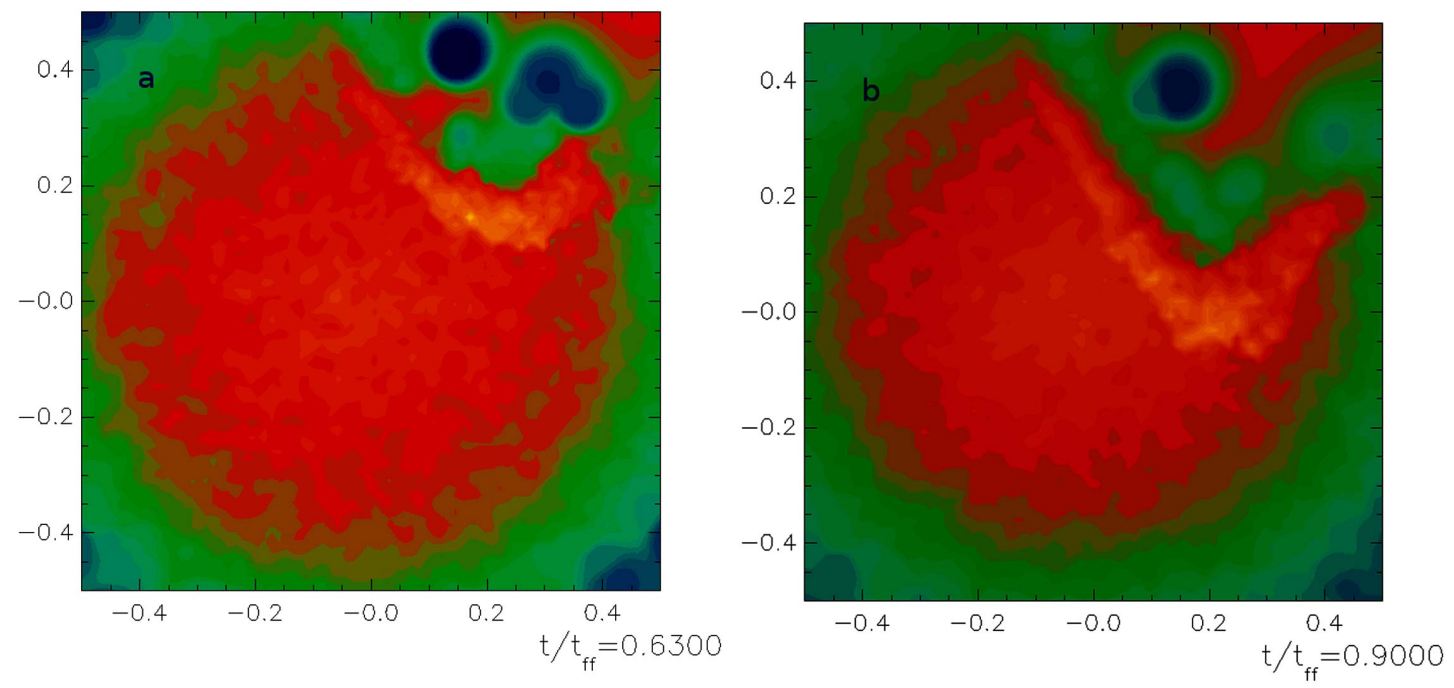

Fig. (9). Contd... 

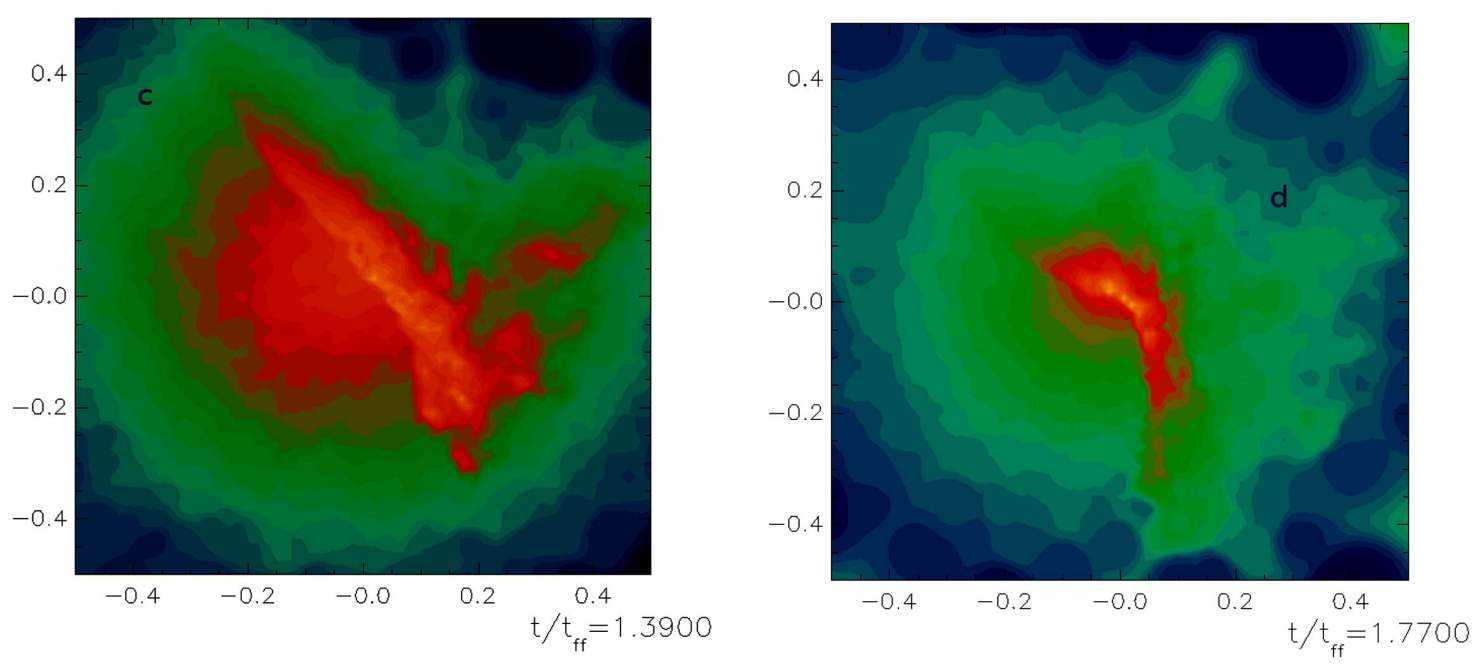

Fig. (9). Iso-density plot for a thin slice of matter parallel to the $X Y$ plane illustrating the collision process of the simulation $0 \mathrm{~b} \sim 2$.

latter the opposite southern side of the colliding region in the target core geometry, is still visible without any changes.

\subsection{The Models $\mathrm{HO}-2$ and $\mathrm{Ob}-2$}

The impact velocity has been increased in these models to almost the double of what was previously used. Then there is a significant increase in the peak density of the models $H O-2$ and $O b-2$, even above the target core average density value, due to a strong wave front. The jump in the peak densities is much bigger than those observed for previous models, see Fig. (3). A well defined bow formed in the interface region prevails.

As expected, the structural changes in the resulting gas core are more evident than ever, as it can be appreciated in Figs. (8 and 9). Following the panels of these Figures, the motion of the wave front itself is easily appreciated. The rotational motion of the initial core has been almost entirely destroyed, leaving behind a region where the turbulent motion of the mixed gas can be seen.

The most important difference between these models can be observed in the results between a head-on collision and with its oblique partner collision, as it can be seen in the two panels of the last row in Figs. (8 and 9). The gas bullet still goes through the target core, leaving behind a trail of corelets in a turbulent environment; for the latter case shown in the right panel, the gas bullet has already gone through, tearing a portion of gas from the target core.

\subsection{The Development of Hydrodynamical Instabilities}

The bow shown in the first panel of Fig. (8) for model $H O-2$ at $t / t_{f f}=0.63$ shall be more closely considered. A zoom into this region is plotted in Fig. (12), showing the particles' velocity field in the wave front. The formation of small bumps can be observed in Fig. (12) in the interface between the particles coming in the wave front and those in circular motion still belonging to the target core. These small bumps are located along the bow at irregular separations, giving the impression of small gas bumps chained along the bow with a finger-like structure. Furthermore, by zooming in the electronic version of this plot, it is possible to see the formation of small velocity vortices, mainly in the arc side arms of the bow (in the lateral regions of the bow).

Let us consider the velocity field corresponding to the last snapshot iso-density plot obtained for model $\mathrm{HO}-2$, shown in the fifth panel of Fig. (10). Here we see the formation of gas velocity vortices in the bullet southern region, where a particles flow is being expelled by the wave front. It is clearly seen that the bullet gas is accreting particles which are still showing the rotational motion of the target core.

All these small structures, including both the small gas bumps and the velocity vortices, can be recognized as hydrodynamical instabilities developing in the interface between the colliding cores. Thus, the small bumps are formed due to the density gradient in the interface region: these bumps indicate the presence of Rayleigh-Taylor instability (RTI). Meanwhile, the small velocity vortices are due to the existence of velocity gradient in the interface, showing the presence of the Kelvin-Helmholtz instability (KHI).

It must be emphasized that all hydrodynamical instabilities observed in these plots for the $\mathrm{HO}-2$ model are also present in the $H O-1$ and $O b-1$ models, although they are not as evident.

\subsection{The Integral Properties}

Another very important objective of this paper is to report on the integral properties of the gas cores after the occurrence of a collision, especially, to measure what are the changes in these properties, from the begining to the final state of the system. The time evolution of these integral 

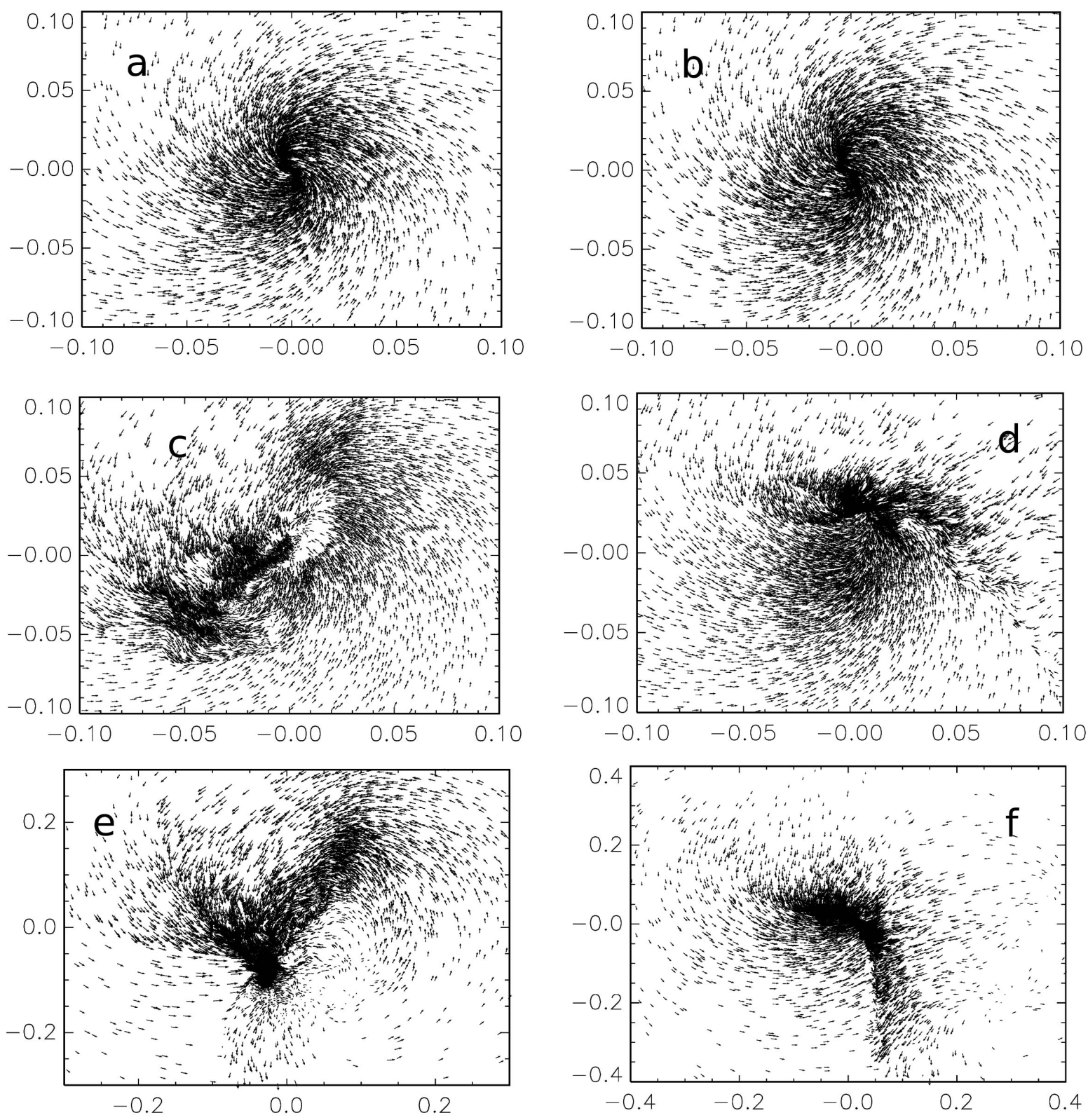

Fig. (10). Plot illustrating the velocity distribution for the last snapshot of the following runs (a) $\mathrm{HO} \sim 0$; (b) $\mathrm{Ob} \sim 0 ;(\mathbf{c}) \mathrm{HO} \sim 1 ;(\mathbf{d}) \mathrm{Ob} 1$; (e) $\mathrm{HO} \sim 2$ and (f) $\mathrm{Ob} \sim 2$.

properties is also very important, as they characterize the changing physical state of the system under consideration, see for instance, [27].

The results obtained by the application of the calculation procedure explained in Section 3.4, to the first and to the last snapshots of each simulation, are shown in Table 3. Column 1 is the label of the model. The time in which the energy ratios are calculated is indicated in column 2 . The calculated energy ratios $\alpha, \beta$ and $\beta_{r}$, as defined previously, are presented in columns 3,4 and 5. Finally, in the last two columns, the result of the sums $\alpha+\beta$ and $\alpha+\beta_{r}$ are shown, which are indicators of the state of the system by means of the virial theorem, as expressed in Section 2.2.
As a complement to the results in Table $\mathbf{3}$, the time evolution of the calculated energy ratios is shown in Figs. (13, 14 and 15).

An approximation to the temporal evolution of the energy ratios of the target core evolving as an isolated system is shown in Fig. (13). The sense in which the curves are formed is indicated by the arrows in these plots. It can be observed that the target core goes quickly to a stage of maximum kinetic energy ratio $\beta$, which also shows itself in a maximum of the rotational kinetic energy ratio $\beta_{r}$. This maximum is produced as a consequence of the core's initial rotation and the conservation of angular momentum during all the evolution. So, by the end of this first stage of 

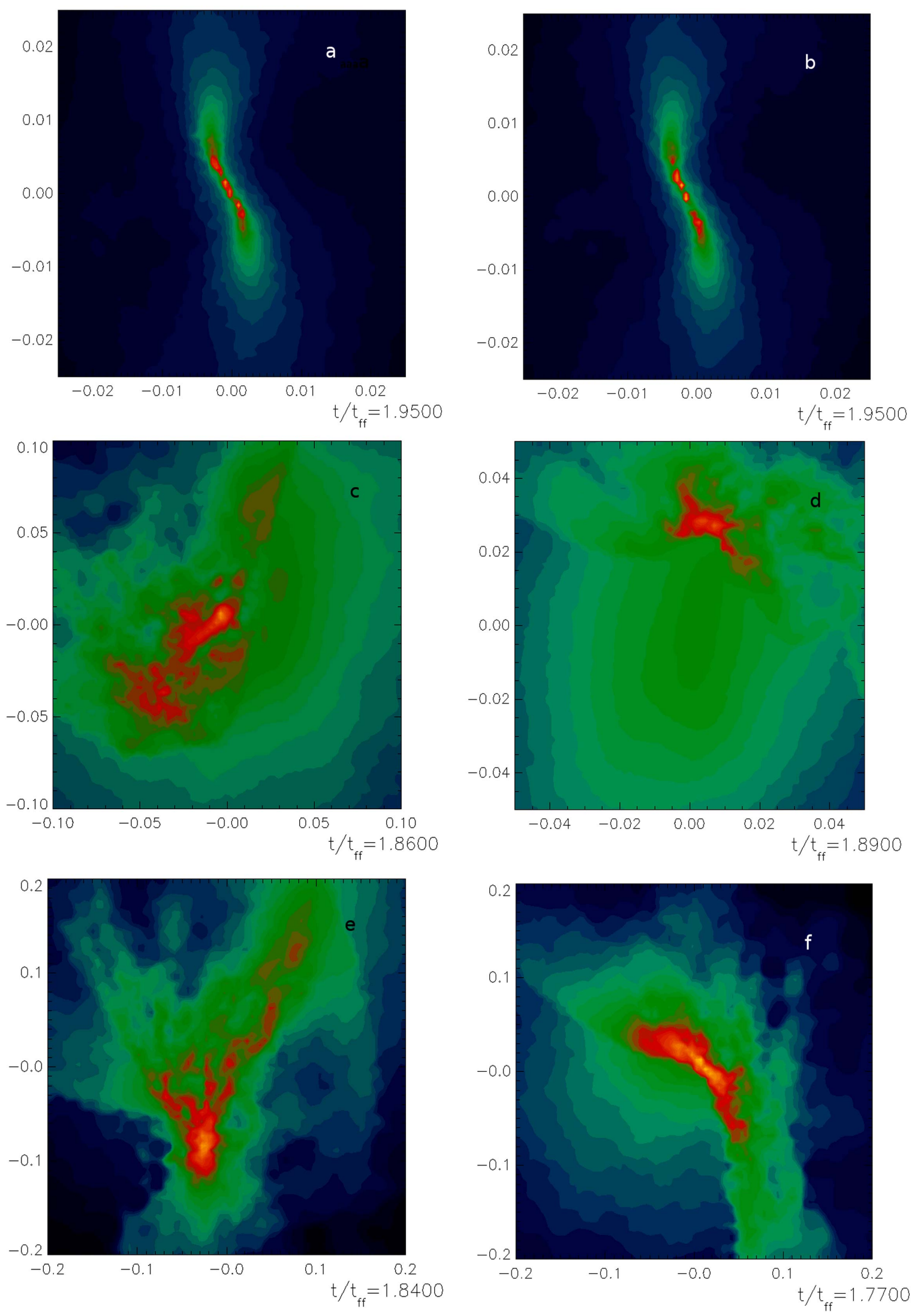

Fig. (11). Zoom in of the central region of the last snapshot of runs (a) $\mathrm{HO} \sim 0$; (b) $\mathrm{Ob} \sim 0$; (c) $\mathrm{HO} \sim 1$; (d) $\mathrm{Ob} \mathrm{1;} \mathrm{(e)} \mathrm{HO} \sim 2$ and (f) $\mathrm{Ob} \mathrm{2.}$ 
Table 3. Integral properties for both the initial and final state of the system.

\begin{tabular}{|c|c|c|c|c|c|c|}
\hline Model & $t$ & $|\alpha|$ & $|\beta|$ & $\left|\beta_{r}\right|$ & $|\alpha|+|\beta|$ & $|\alpha|+\left|\beta_{r}\right|$ \\
\hline \multirow[t]{2}{*}{ Target Cloud } & 0 & 0.261620 & 0.159898 & 0.159882 & 0.42 & 0.42 \\
\hline & $5.69 * t_{f f c}$ & 0.163084 & 0.374863 & 0.163744 & 0.53 & 0.32 \\
\hline HO-0 & 0 & 0.264115 & 0.156913 & ---- & 0.42 & ---- \\
\hline \multirow[t]{2}{*}{ HO-1 } & 0 & 0.264115 & 0.322109 & ---- & 0.58 & ---- \\
\hline & $1.49 * t_{f f}$ & 0.204720 & 0.343520 & 0.170658 & 0.54 & 0.37 \\
\hline $\mathrm{HO}-2$ & 0 & 0.264115 & 0.818150 & ---- & 1.07 & ---- \\
\hline \multirow[t]{2}{*}{ Ob-1 } & 0 & 0.264339 & 0.332722 & ---- & 0.59 & ---- \\
\hline & $1.51^{*} t_{f f}$ & 0.206420 & 0.311773 & 0.138507 & 0.51 & 0.34 \\
\hline \multirow[t]{2}{*}{$\mathrm{Ob}-2$} & 0 & 0.264339 & 0.860218 & ---- & 1.12 & ---- \\
\hline & $1.77 * t_{f f}$ & 0.153506 & 0.371069 & 0.155077 & 0.52 & 0.30 \\
\hline
\end{tabular}

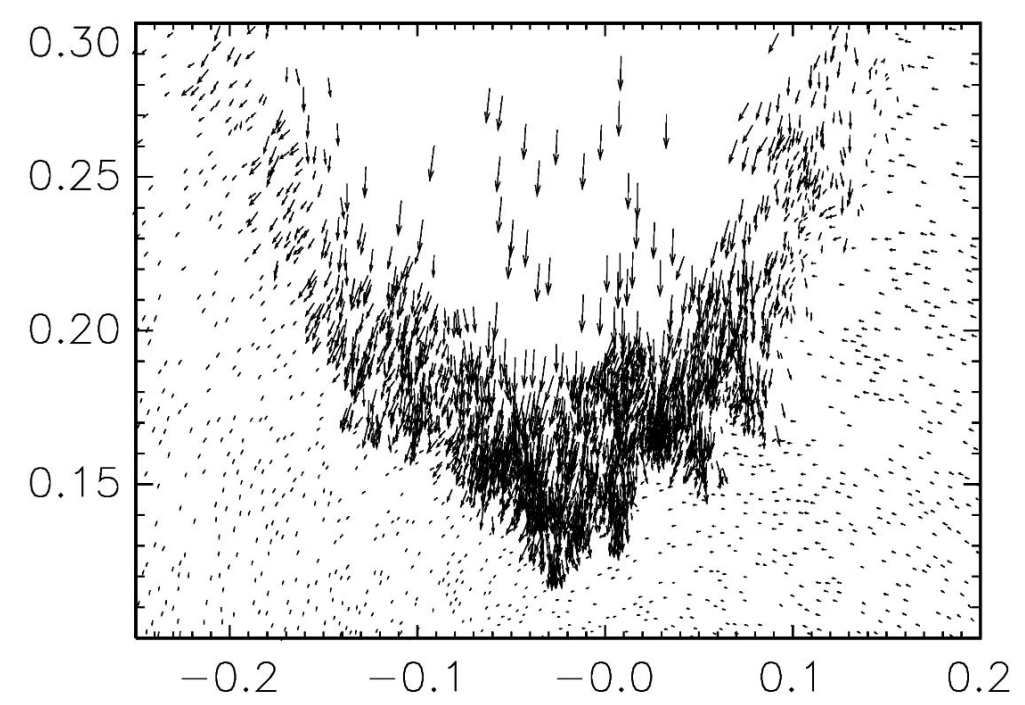

Fig. (12). The shock front for model HO-2 corresponding to the panel 3 with $t=t_{f f}=0: 63$ at the left side of the middle row of Fig. $(\mathbf{8})$.

evolution, the core center has acquired the form of a flattened disk that spins faster than the rest of the gas located at the target core outer regions.

The core's flattening proces is interrupted because of the dynamical equilibrium is reached by the gas thermal pressure, mainly in the vertical direction. The kinetic energy of the core returns to a value closer to the virial line.
The width of the flattened disk is determined by this dynamic equilibrium. The particles located near the central region are being strongly accreted by the disk, increasing the potential energy of those particles that have already fallen into the disk, while becoming faster than those particles still falling. As a manifestation of this accretion phenomenon, it can be seen in Fig. (13) that $\alpha$ is decreasing while $\beta$ is increasing. 

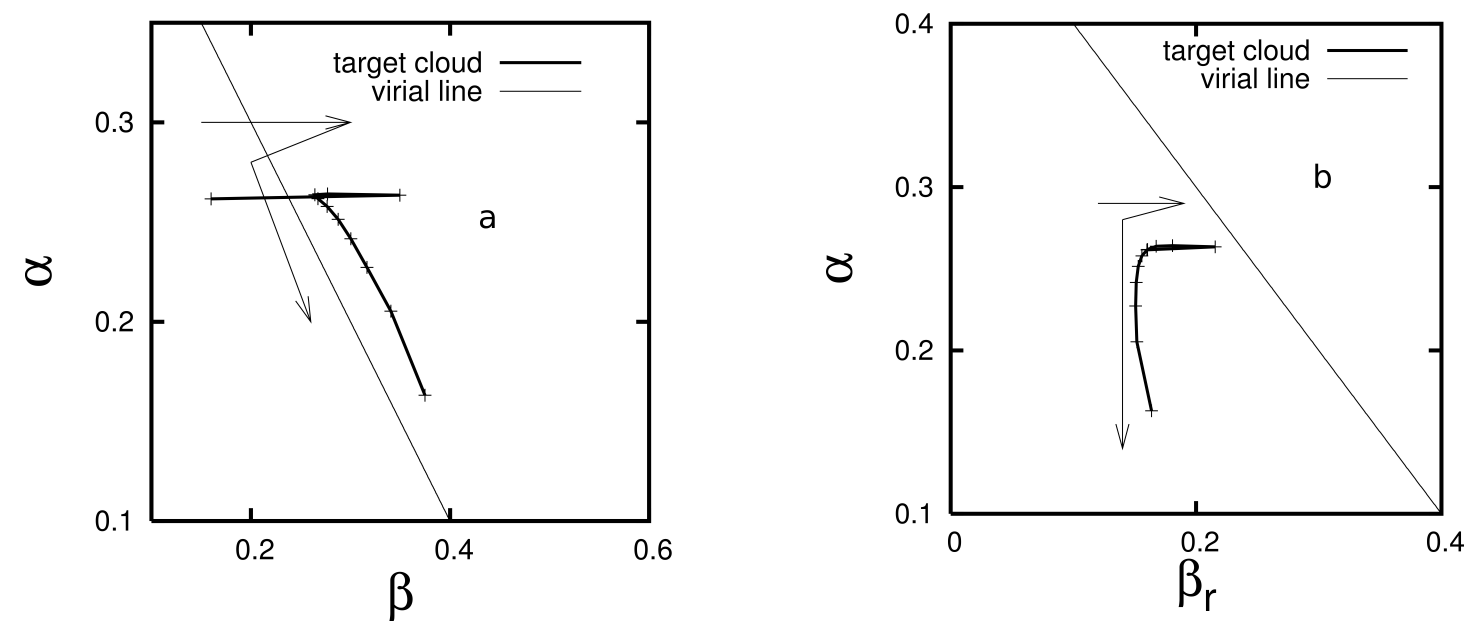

Fig. (13). Integral properties for the target cloud (left) with kinetic energy and (right) rotational energy.
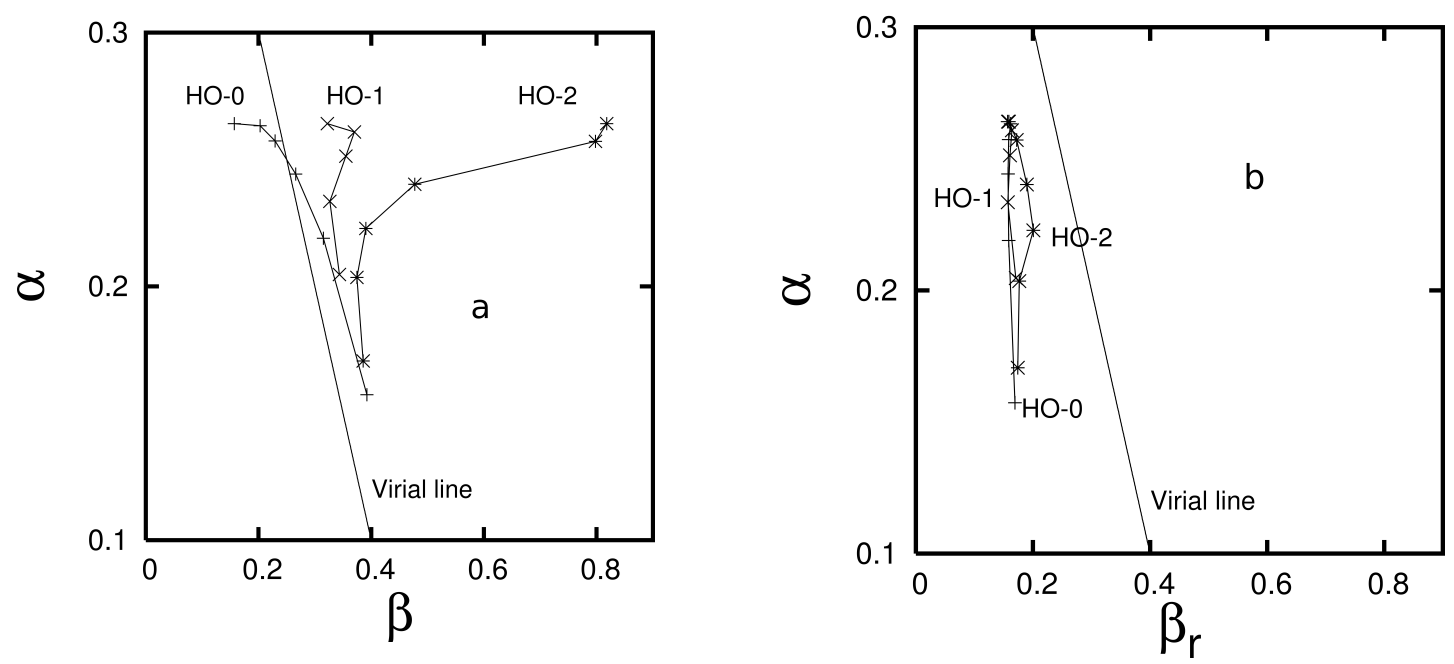

Fig. (14). Integral properties for head-on collisions (left) with kinetic energy and (right) rotational energy.

All the simulation particles are included for the calculation of the integral properties, as explained in Section 3.4. Only a very small fraction of the total number of particles is near or inside the densest center of the core. Therefore, it can be seen that most of the particles in the outermost region are still rotating as a rigid body.

If the core's gravitational collapse could have been followed further, the physical expectation for the subsequent evolution of the core would show both curves in the $\alpha$ vs. $\beta$ and $\alpha$ vs. $\beta_{r}$ planes would approach to the virial line, but each one of the curves is going from their own side, as seems to be pointed out by the curvature of each curve in Fig. (13).

Consequently, it would be expected that, by the end of the collapse of the core, the curve of the evolution of the energy ratios in the $\alpha$ vs. $\beta_{r}$ plane would show a concave upwards curve, and its southern ending point would be stretched, reaching a higher level of evolution.

It can be immediately appreciated in Figs. (14 and 15) that the initial kinetic energy ratio has a high value due to the translational velocity contribution of the particles in the bullet. As the collision takes place, and as the particles interact by means of the artificial viscosity included in the code, the excess of kinetic energy is transformed into heat.

Let us compare the plots of the evolution of the energy ratios for the collision systems shown in Figs. (14 and 15), with those obtained for the target core as an isolated system showing Fig. (13). It may be concluded that it is the rotational motion of the target core which survives and passes on its energy ratios to the mixed gas core for all the collision cases considered in the present paper.

\subsection{The Time Evolution of the System Energies}

The evolution of the relevant energies for the gravitational collapse of the target core as an isolated system is shown in the left panel of the top row of Fig. (16). As expected, the mechanical energy $M E$ is always negative, indicating that the core is collapsing. The thermal energy $I E$ remains almost constant until $80 \%$ of the full evolution of 

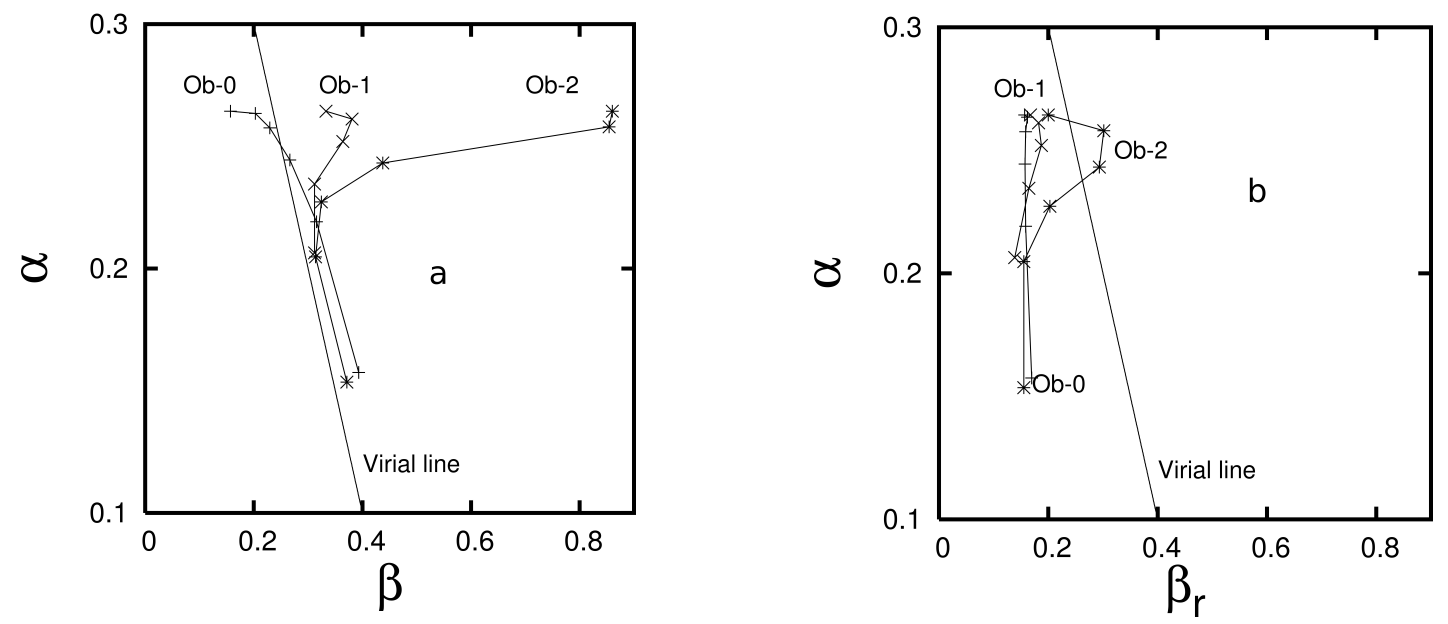

Fig. (15). Integral properties for oblique collisions (left) with kinetic energy and (right) rotational energy.

the core's collapse has been reached. Afterwards, a slight increase is seen, indicating that the potential energy $P E$ acquired by the core collapse is being transformed to thermal energy. The conservation of total TE energy is poor at the final stage of evolution.

The main objective here is to compare the energies evolution of the isolated target core with those of the mixed gas core after collision. For this purpose, the coordinate origin has been changed to the center of the target core in order to calculate its energetic evolution during the collision. Those particles located within $1.2 R_{0}$ from its center $\vec{X}_{T C}$ have been considered, consequently, all the particles belonging to the bullet core for the calculation of the energies have been ignored, at least during the first stages of evolution of each colliding model.

The results of this calculation are shown in the three additional plots of Fig. (16). The thermal energy (TE) evolution of the gas mixed core is shown on the right panel of the top row. In the bottom left panel of Fig. (16), the kinetic and potential energies of all of the collision systems are shown. It can be seen that the isolated core potential energy $P E$ is the main negative contribution to the potential energy of the collision model.

As it can be seen in the bottom right panel of Fig. (16) the evolution of the mechanical energy $(M E)$, of all the collision systems is always negative, indicating the bound nature of the mixed gas core; the kinetic energy increase is noticeable due to those bullet core particles just entering into the target core. As a result, it is worth mentioning that the surplus initial kinetic energy supplied by the bullet core is mainly transformed into potential energy of the mixed gas core as the collision takes place. A curve labeled TE - TC has been included in this plot: it corresponds to the total energy of the target core when evolving as an isolated system. It is interesting to note that the total energy of all the collision systems are below the curve $T E-T C$.

\section{DISCUSSION}

A few models of a collision system composed of two dissimilar cores have been considered in the present study, in order to see how collisions can disturb the initial physical state of a rotating core. Additionally, the physical properties of the newly formed mixed gas core resulting from the interpenetration of a bullet core gas into the target core have also been determined in the present study. Some of the physical features observed in the present investigation shall be highlighted in the following subsections.

It is known that there are several physical phenomena which compete to exert more influence on the collision process: self-gravity, tidal torques and the frontal wave. The occurrence and influence of these physical factors on the results of a simulation are mainly regulated by the value of the approach velocity of the colliding cores.

Thus, in a collision system with a low approach velocity, self-gravity dominates the early evolution of the colliding cores with the main effect being a decrease in the size of each core. When the two cores finally collide, a wave front at the interface of both collapsing clumps is formed.

\subsection{Comparison with Paper I and with Some Previous Papers}

Recal that in Paper I, the collision systems studied were composed of two identical cores, having the same mass and radial density profile; the same initial rotation, and with both cores having the same translation velocity, each in the opposite direction. It should be noted that the same prescription and values for the artificial viscosity have been used for the calculations of this paper as for those of Paper I. The colliding cores evolution code and initial configuration in Paper I, as well as those for the target core in the present paper, are also the same.

Regardless of all these technical similarities, there are some very important physical differences in the results of 

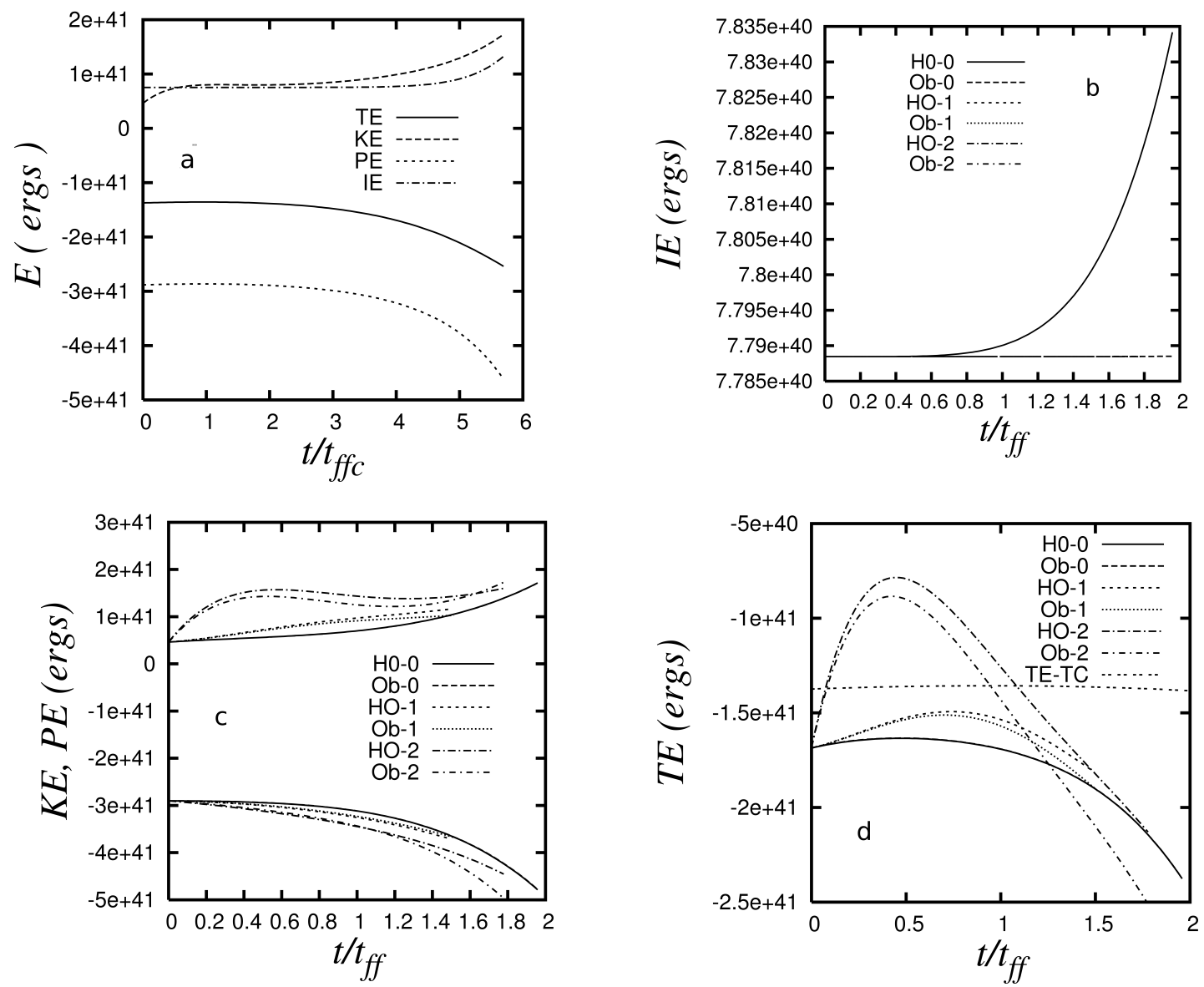

Fig. (16). Time evolution of energies for the isolated core (left top) and for the collision system (right top and the bottom row). The abbreviations are as follows: the mechanical energy $\$ M E \$$ is defined as $\$ M E=K E+P E \$$ where $\$ K E \$=$ total kinetic energy and $\$ P E \$=$ total potential energy; \$IE $\$=$ total thermal energy and $\$$ TE $\$=$ total energy defined as $\$$ TE=ME+IE $\$$. The curve labeled as TE-TC in the fourth panel stands for the total energy of the target core as was plotted in the first panel.

these two papers. For instance, the cores' interpenetration was always observed in the present paper, whereas an interpenetration was observed in Paper I only for models with a very high impact velocity. Meanwhile, for the models with an impact velocity in a range similar to that of models of this paper, no interpenetration at all was seen in Paper I. Some speculations as to the dynamical reasons that could explain this difference in the occurrence of an interpenetration follows.

A filament (or gas bridge) connecting the two shrunken cores is produced by the wave front in the low impact velocity simulations of Paper I. The two remaining clumps act upon the particles in the bridge by exerting a gravitational pull toward their respective centers. As the clumps have the same mass, the tidal pull magnitude is almost the same, but each in the opposite direction.

Moreover, it must be taken into account that the direction of the initial rotation of the cores provides inertia to each collapsing core in the colliding system, with a tendency to lengthen the bridge of dense particles by its ends. An interpenetration of the cores in this kind of models is thus avoided by the combination of tidal forces and the initial geometry of the colliding systems, as was previously discussed in Paper I.

The gravitational field that the target core exerts on the bullet core in all the models carried out in the present study, irrespective of the impact velocity, are obviously more intense, as its mass is much bigger than the bullet mass. There is also a filament formed in the interface region between the cores, but the filament and the bullet core itself are attracted and eventually engulfed entirely by the target core. Therefore, interpenetration is obviously unavoidable.

It was also shown in Paper I that in a collision system with a sufficiently high impact velocity, the fate of the system is entirely determined by the strong flow of particles originating in the interface of the colliding cores. It was observed that the whole of the colliding cores are completely replaced by a single mixed gas core, forming an elongated and irregular filamentary structure, which increases its size very rapidly as it is continuously fed by the in-falling material coming from the original cores. The resulting mixed core can be considered as the only example of interpenetration of cores observed in Paper I. 
These high impact velocity models are interesting from the star formation point of view, as this kind of high velocity collisions may be the mechanism for the formation of the observed filamentary structure of the interstellar medium (ISM), as [10] has already speculated.

It was seen in the low impact velocity models of Paper I, that the bridge of gas is highly irregular, as many particles are continuously ejected from the colliding interface and moving very fast in the directions perpendicular to the core collision axis. It should be mentioned that this resulting gas configuration is interesting and complex, as it is composed of the filament or bridge of dense particles as well as the two remaining collapsing cores, where there is still the possibility that the formation of a cluster of protostars core hopefully take place, as the late evolution of the colliding system is guided by the ongoing cores gravitational collapse.

It is in this filament where the $K H$ instabilities were observed in both Paper I and in the present paper; there is still the chance that these hydrodynamical instabilities can grow further in this filamentary structure and eventually lead to the formation of a protostar, as [28] has previously speculated.

By comparing the results obtained in Paper I with the calculations reported by [10] and [28], we conclude that having considered a collapsing and rotating initial core was crucial for obtaining the complex gas configuration resulting from the collision systems. These gas configurations will likely become the initial sites where protostar formation may eventually initiate.

The formation and evolution of the bow, as was observed by [11], is also observed here. However, it appears that the initial rotation given to the cores plays a very important role in the development of the hydro-dynamical instabilities.

As mentioned earlier in this section, we may conclude that collisions between dissimilar cores are less interesting than those collision systems composed of two similar cores, at least from the protostar formation perspective.

\subsection{Filament Fragmentation and other Physical Properties}

There is an additional very important result found by studying the simulations of Paper I, which fortunately we meet again in this paper, irrespective of the different collisions produced due to the very small mass of the onrushing core.

For the oblique collision model of Paper I with $b=R_{0}$ and $V_{a p p}=2.74 \times c_{0}$, which was labeled as model $M 2$, it was found that a filament developed in the central region of each core, showing a clear tendency to fragment by forming small knots along the filament. This behavior contrasts with the fate of the filament developed by the initial core if it was evolving as an isolated system, in which it showed no tendency to fragment.

It was then concluded that the fragmentation of the filaments in model $M 2$ was a consequence of the collision.
It is observed in this paper that the same behavior occurs in the central region of the target core for models $\mathrm{HO}-0$ and $O b-0$, irrespective of the value of the impact parameter $b$, as can be appreciated by comparing the first two plots of Fig. (11) with Fig. (2).

It is noteworthy that fragmentation of the filaments has been recently reported by [29], who presented a study of dense structures in the L1495 filament in the Taurus molecular core. Highly fragmented sub-structures can be seen in the filaments; some of these sub-structures are potential star forming sites as they are indeed capable of further collapse. A possible mechanism for forming such filamentary structures with a dense substructure is provided here by these simulations.

It should be noticed that the final density profile curves obtained for models with significant impact velocity show a clear tendency to become flat (at least they do not have a steep slope) in the innermost central region of the core while falling off for higher radii. This radial behavior is in better agreement with core density structure observations. It must be emphasized here that this change in the radial density structure from uniform to centrally condensed, was indeed induced by the occurrence of the collision.

[30] have performed calculations by colliding two identical polytropes with their surfaces barely touching each other, including self-gravity and viscosity, with the purpose of testing the conservation of energy in codes based on the SPH technique. They have found that the errors in the nonconservation of energy can be as high as $10 \%$ when the $\nabla h$ terms are not included in the equation of motion of the particles.

Since the public version of the Gadget 2 code used here does not include the $\nabla h$ terms, we are in the worse nonconserving energy scenario. Consequently, large errors in the system energy evolution are then to be expected. The time evolution of the energies in the collision models, have been included in Fig. (16). It is observed that the expected errors are more evident in the last collapsing stage, but in spite of this, something useful has been taken from the calculations described in this paper.

\section{CONCLUDING REMARKS}

Molecular core collisions may be very common in the Interstellar Medium. It has been mentioned by [31] and [8] that it is more likely that a colliding system will result in the disruption and dispersal of the involved cores, with no chance of forming protostars. But there is still the possibility that small cores collide forming larger gas associations by means of coalescence and eventually, if the gravitational collapse proceeds further, a low mass protostar formation would be possible.

Even though this paper has been limited to considering only highly idealized collision systems, it has been possible to capture and display some of the essential physics of a collapsing core (the target), that is penetrated by a denser gas 
core (the bullet). Although, the parameter space of the collision systems for the models of Paper I was better explored than in the present paper, both investigations have always focused on simulations avoiding disruption and total dispersion of the colliding cores.

The results of this paper are summarized as follows:

- The observation that collisions favor fragmentation in molecular cores is a very important one, because each of the dense gas knots formed along the filament in the central core of the colliding core, could eventually form a proto-star.

- Less striking but by no means less important, is our observation made about the fifth panel of Fig. (11), which shows that high density clumps are formed behind the wave front left by the bullet along its way digging into the target core. There is still the chance that these condensations of gas may end up as proto-stars.

More collision models from which the subtleties of the relation between the physical phenomena of collision and protostar formation can be pointed out as needed, see also [32] and [33].

\section{CONFLICT OF INTEREST}

The authors confirm that this article content has no conflicts of interest.

\section{ACKNOWLEDGMENTS}

GA. would like to thank ACARUS-UNISON for the use of their computing facilities in the making of this paper.

\section{REFERENCES}

[1] Mookerja SK, Ghosth TN. Distribution of cold dust in orion A and B. ApJ 2000; 120: 1954-62.

[2] Thronson HA, Jr, Harper DA, Bally J, et al. The orion star forming region: far-infrared and radio molecular observations. Astron $\mathbf{J}$ 1986; 91(6): 1350-489.

[3] Egusa F, Koda J, Scoville. Molecular gas evolution across a spiral arm in M51, N. Astron J 2011; 726: 85.

[4] Norman C, Silk J. Clumpy molecular clouds - a dynamic-model self-consistently regulated by T-Tauri star formation. ApJ 1980; 238: 158-74.

[5] Loren RB. Colliding clouds and star formation in NGC 1333. ApJ 1976; 209: 466-88.

[6] Burkert A, Alves J. The inevitable future of the starless core barnard 68. ApJ 2009; 695: 1308.

[7] Bekki K, Beasley M, Forbes D, Couch WJ. Formation of star clusters in the Large Magellanic cloud and Small Magellanic cloud. I. Preliminary results on cluster formation from colliding gas clouds. ApJ 2004; 602: 730

[8] Kimura T, Tosa M. Collision of clumpy molecular clouds. Astron Astrophys 1996; 308: 979.

[9] Arreaga-Garcia G, Klapp-Escribano J, Saucedo-Morales J. Simulations of colliding uniform density $\mathrm{H} 2$ clouds. Int J Astron Astrophys 2014; 4(1): 192-220.
[10] Anathpindika S. Supersonic cloud collision. Astron Astrophys 2009; 504: 437-50.

[11] Anathpindika S. Collision between dissimilar clouds: stability of the bow-shock, and the formation of pre-stellar cores. MNRAS 2010; 405: 1431-43.

[12] Chapman S, Pongraic H, Disney M, Nelson A, Turner J, Whitworth A. The formation of binary and multiple star systems. Nature 1992; 359(2): 207-10.

[13] Bergin E, Tafalla M. Cold dark clouds: the initial conditions for star formation. Annu Rev Astro Astrophys 2007, 45: 339.

[14] Myers PC, Benson PJ. Dense cores in dark clouds. II - NH3 observations and star formation. Astrophys J 1983; 266: 309.

[15] Arreaga-Garcia G, Klapp J, Sigalotti LG, Gabbasov R. Gravitational collapse and fragmentation of molecular Cloud Cores with GADGET-2. ApJ 2007; 666: 290-308.

[16] Arreaga-Garcia G, Saucedo J, Duarte R, Carmona J. Hydrodynamical simulations of the non-ideal gravitational collapse of a molecular gas cloud. Rev Mex Astron Astrophys 2008; 44: 259-84.

[17] Gingold RA, Monaghan JJ. Smooth particle hydrodynamics:theory and applications to non-spherical stars. MNRAS1977; 181: 375-89.

[18] Balsara D. von Neumann stability analysis of smooth particle hydrodynamics--suggestions for optimal algorithms. J Comput Phys 1995; 121: 357-72.

[19] Springel V, Yoshida N, White DM. GADGET: a code for collisionless and gasdynamical cosmological simulations. New Astron 2001; 6: 79-117.

[20] Boss AP, Fisher RT, Klein R, McKee CF. The jeans condition and collapsing molecular cloud cores: filaments or Binaries ? ApJ 2000; 528: 325-35.

[21] Spitzer L. Physical processes in the interstellar medium. USA: Wiley 1978.

[22] Gilden DL. Clump collisions in molecular clouds - gravitationalinstability and coalescence. ApJ 1984; 279: 335-49.

[23] Truelove JK, Klein RI, McKee CF, Holliman JH, Howell LH, Greenough JA.The jeans condition: a new constraint on spatial resolution in simulations of isothermal self-gravitational hydrodynamics. ApJ 1997; 489: L179-L183.

[24] Bate MR, Burkert A. Resolution requirements for smoothed particle hydrodynamics calculations with self-gravity. MNRAS 1997; 288: 1060-72.

[25] Bodenheimer P, Burkert A, Klein RI, Boss AP. Multiple Fragmentation of Protostars. In: Mannings VG, Boss AP, Russell SS, Eds. Protostars and Planets IV. Tucson: University of Arizona Press 2000; pp. 675-70.

[26] Sigalotti LG, Klapp J. Gravitational collapse and fragmentation of molecular cloud cores. Int J Mod Phys D 2001; 10: 115-211.

[27] Arreaga-Garcia G, Saucedo J. Physical effects of gas envelopes with different extension on the collapse of a gas core. Rev. Mex. Astron. Astrophy. 2012; 48: 61-84.

[28] Anathpindika S. Supersonic cloud collision - II. Astron Astrophys 2009; 504: 451-60.

[29] Quanz SP, Alves J, Roman-Zuniga CG, et al. Star formation in the Taurus filament L1495: From Dense cores to stars A\&A (Astronomy and Astrophysics) 2010; 725: 1327-1336. arXiv- eprint astro-ph/1010.2755.

[30] Nelson RP, Papaloizou CB. Variable smoothing lengths and energy conservation insmoothed particle hydrodynamics. MNRAS 1994; 270: 1-20.

[31] Whithworth AP, Pongracic H. In: Fragmentation of molecular clouds and star formation. Falgarone E, Boulangeer F, Duvert G, Eds. Kluwer: International Astronomical Union 1991; p. 523.

[32] Kumai Y, Basu B, Fujimoto M. Formation of globular-clusters from gas in large-scale unorganized motion in galaxies. ApJ 1993 404: 144-61.

[33] Fujimoto M, Kumai Y. Star clusters driven to form by strong collisions between gas clouds in high-velocity random motion. Astron J 1997; 113: 249-63.

(C) Arreaga-García and Saucedo-Morales; Licensee Bentham Open.

This is an open access article licensed under the terms of the Creative Commons Attribution Non-Commercial License (http://creativecommons.org/licenses/by-nc/3.0/) which permits unrestricted, non-commercial use, distribution and reproduction in any medium, provided the work is properly cited. 